Article

\title{
An Efficiently Decoupled Implicit Method for Complex Natural Gas Pipeline Network Simulation
}

\author{
Peng Wang ${ }^{1}$, Shangmin Ao ${ }^{1}$, Bo Yu ${ }^{1, *}$, Dongxu Han ${ }^{1}$ and Yue Xiang ${ }^{2}$ \\ 1 School of Mechanical Engineering, Beijing Key Laboratory of Pipeline Critical Technology and Equipment \\ for Deepwater Oil \& Gas Development, Beijing Institute of Petrochemical Technology, Beijing 102617, China; \\ wangp@bipt.edu.cn (P.W.); aoshangmin@126.com (S.A.); handongxubox@bipt.edu.cn (D.H.) \\ 2 Research Center of Cloud Simulation and Intelligent Decision-making, Tsinghua Sichuan Energy Internet \\ Research Institute, Chengdu 610042, China; cupxy@foxmail.com \\ * Correspondence: yubobox@vip.163.com; Tel.: +86-01-8129-2805
}

Received: 1 March 2019; Accepted: 18 April 2019; Published: 22 April 2019

check for updates

\begin{abstract}
The simulation of a natural gas pipeline network allows us to predict the behavior of a gas network system under different conditions. Such predictions can be effectively used to guide decisions regarding the design and operation of the real system. The simulation is generally associated with a high computational cost since the pipeline network is becoming more and more complex, as well as large-scale. In our previous study, the Decoupled Implicit Method for Efficient Network Simulation (DIMENS) method was proposed based on the 'Divide-and-Conquer Approach' ideal, and its computational speed was obviously high. However, only continuity/momentum Equations of the simple pipeline network composed of pipelines were studied in our previous work. In this paper, the DIMENS method is extended to the continuity/momentum and energy Equations coupled with the complex pipeline network, which includes pipelines and non-pipeline components. The extended DIMENS method can be used to solve more complex engineering problems than before. To extend the DIMENS method, two key issues are addressed in this paper. One is that the non-pipeline components are appropriately solved as the multi-component interconnection nodes; the other is that the procedures of solving the energy Equation are designed based on the gas flow direction in the pipeline. To validate the accuracy and efficiency of the present method, an example of a complex pipeline network is provided. From the result, it can be concluded that the accuracy of the proposed method is equivalent to that of the Stoner Pipeline Simulator (SPS), which includes commercially available simulation core codes, while the efficiency of the present method is over two times higher than that of the SPS.
\end{abstract}

Keywords: natural gas; pipeline network; continuity/momentum and energy equations coupled; efficient simulation

\section{Introduction}

As a high quality and clean fossil fuel, natural gas plays an important role in global industry and economy [1]. Pipelines are the primary means by which the natural gas is transported. As more and more cities are using natural gas as the main energy source, the pipeline network is becoming extraordinarily complex. The characteristic of a complex topology has created many challenges for the design, monitoring, and operating of the pipeline network. The simulation of the natural gas pipeline network allows us to predict the behavior of a gas network system under different conditions. By matching the simulator's output with measured information from Supervisory Control and Data Administration (SCADA) systems, the gas pipelines can be estimated in real-time [2], the historical conditions can be reviewed, and the unexpected transient conditions can be analyzed [3]. 
Such predictions and analyses can be effectively used to guide decisions regarding the design and operation of the real pipeline network system. For example, Gssco improved his pipeline capacity with the help of a commercial tool known as the Pipeline Modeling System (PMS) [4].

Depending on the gas flow characteristics in the network system, there are two states in which the simulation can be: steady simulation (static simulation) and unsteady simulation (transient simulation). Steady simulation does not take into account the gas flow characteristics' variations over time. The goal of steady simulation is usually to compute the nodes' pressures, the nodes' loads, and the pipes' flow rates for the network design. The pressures at the nodes and the flow rates in the pipes must satisfy the general flow equations; the lodes' loads must fulfill the first Kirchhoff's law or Node formulation and the pressure drop around any closed loop must fulfill the second Kirchhoff's law or Loop formulation. The steady simulation was explained in detail by Osiadacz [5], and interested readers can refer to it. Unsteady simulation considers the facts that gas flow characteristics are mainly functions of time. In fact, the gas flow in a pipeline is actually a transient process, while a steady state is rare in practice. Thus, unsteady simulation is more practical for the operations of a real pipeline network. However, the mathematical model of unsteady simulation is a system of partial differential equations (PDEs) of mass conservation, momentum conservation, and energy conservation. The mathematical model is usually solved by numerical methods [6-15], and the frequently used numerical methods are method of characteristics (MOC), finite difference methods (FDM), and finite element methods (FEM). MOC firstly reduces PDEs to a family of ordinary differential equations (ODEs) along the characteristic curves, and the ODEs are then solved along the characteristic curves to obtain the flow and thermodynamic parameters. FDM firstly divides the long pipeline into many short sections, then converts PDEs into a system of difference Equations (DEs) on these small sections, and lastly solves DEs by matrix algebra techniques. FEM firstly divides the entire pipeline into many small sections that are so-called finite elements, then uses variational methods to derive the simple equations for these finite elements, and lastly solves the larger system of simple equations by minimizing an associated error function. Thorley and Tiley [16] have provided an excellent literature review of these methods, and interested readers can refer to it.

Among these methods, the implicit finite difference method is one of the most widely applied methods because its time step is not restricted by the stability criterion [6,17-19]. This means that the time step of the implicit finite difference method can be very large, which is very useful for simulating long-term transient flow in a natural gas pipeline. However, in the implicit finite difference method, one system of large-scale nonlinear discretized Equations needs to be solved [20]. When working with large-scale pipeline networks, its efficiency could be low and unsatisfactory, especially on a personal computer.

To improve the computational speed of the implicit finite difference method, a series of research projects have been carried out. Kiuchi [17,18] and Wylie et al. [19] directly ignored the convective inertia term in the governing Equations of a pipeline to avoid nonlinear equations. However, this process could reduce the accuracy of simulation [21]. Luskin [22] proposed a linearized method where the nonlinear governing equations were linearized about the previous time step based on the Taylor expansion. Zheng et al. [23] found that the linearized method could improve the computational speed by over five times. Barley [24] and Helgaker and Ytrehus [25] put forward a decoupled solution strategy, in which the continuity/momentum equations (flow equations) and energy equation (thermodynamics equation) were solved alternatively. This strategy could further increase the computational speed by about $20 \%$. Wang et al. [26] found that the form of flow equations, which took the density and velocity as the solving variables, was the most efficient form. The computational speed was further improved by 50\%. Many researchers, such as Wylie et al. [19] and Stoner [27], have recommended the sparse matrix technique to efficiently solve the large-scale discretized equations of network simulation. Madoliat et al. [13] proposed a novel approach based on intelligent algorithms, such as particle swarm optimization (PSO), for dynamic simulation of a gas pipeline network. These studies have improved the computational speed of natural gas pipeline network simulation to a considerable extent. 
However, in the above studies, all pipelines in the network must be solved simultaneously, and one system of large-scale equations inevitably has to be solved. It is well-known that the computational speed of small-scale equations is faster than that of large-scale equations. Therefore, dividing the network into several pipelines and then solving them one by one is an effective way to further improve the computational speed of natural gas pipeline network simulation. This is the idea of the 'Divide-and-Conquer Approach'. Based on this idea, a fast method for the flow simulation of natural gas pipeline networks was proposed in our previous study [28], called the Decoupled Implicit Method for Efficient Network Simulation (DIMENS). In the DIMENS method, the flow equations of all the multi-pipeline interconnection nodes were firstly solved, and the flow parameters such as pressure and flow rate were known; the pipeline network was then divided into several independent pipelines, and the pipelines were efficiently solved one by one. Thus, this method is more efficient than the commercially available simulation core codes of Stoner Pipeline Simulator (SPS), whose computational speed can represent the highest level in the world.

In our previous work [28], the gas flow was considered isothermal, and the pipeline network was only composed of pipelines. Thus, the DIMENS method was only implemented in flow equations of the simple pipeline network. However, in practical engineering problems, the pipeline network is generally very complex, which includes not only pipelines, but also non-pipeline components, such as compressors, valves, supplies, and demands [12]. What is more, many researchers [24,25,29,30] have shown that the thermodynamic parameters have a major impact on the flow parameters of a pipeline network, and the effect of treating the gas in a non-isothermal manner was extremely necessary for pipeline flow calculation accuracies.

To overcome the above issue, the DIMENS method is extended to the flow and thermodynamic-coupled simulation of the complex pipeline network in this paper. The layout of this paper is as follows. First, the mathematical model of the pipeline network and its discretization is introduced. Second, the main idea of the DIMENS method is reviewed. Third, the implementation process of the extended DIMENS method is given, and the solution procedures of the thermodynamic equations are elaborated. Finally, a numerical case of the complex pipeline network is designed to test the performance of the present method.

\section{Mathematical Model and Discretization}

\subsection{Mathematical Model}

The pipeline network is mainly formed by interconnecting many kinds of components, and the basic components are pipelines, compressors, valves, supplies, demands, and so on [8]. The components of the pipeline network can be classified into four categories, namely, pipelines, non-pipelines, externals, and multi-component interconnection nodes. Therefore, the mathematical model of the natural gas pipeline network in this paper includes four parts: pipeline model, non-pipeline model, multi-component interconnection node model, and boundary conditions.

\subsubsection{Pipeline Model}

For natural gas pipeline network simulation, the flow in the pipeline is generally assumed to be homogeneous equilibrium flow with negligible fluid/structure interactions, and the influence of the non-uniformity of the velocity distribution is neglected. The pipeline model is a set of governing equations of homogeneous, geometrically one-dimensional flow in the pipeline, which consist of the continuity equation, momentum equation, and energy equation. The continuity and momentum equations are so-called flow equations, and the energy equation is a so-called thermodynamic equation. These equations can be written in a general form [24-26,31], as shown in Equation (1), and 50the parameters of the general form are given in Table 1. The detailed process of transformations and the parameter expressions of Equation (1) can be found in Appendix A: 


$$
\frac{\partial \mathbf{U}}{\partial t}+\mathbf{B} \cdot \frac{\partial \mathbf{U}}{\partial x}=\mathbf{F}
$$

Table 1. Parameters in the governing equations.

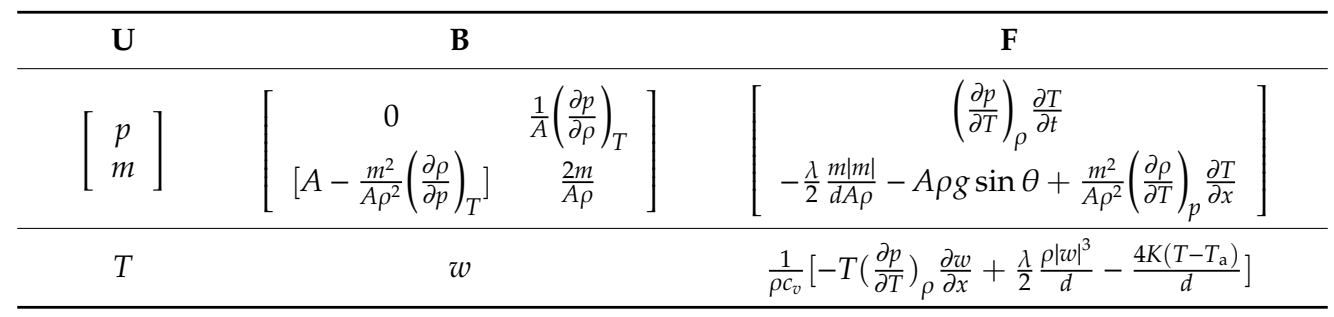

It should be noted that natural gas is a compressible gas, and its thermodynamic properties (pressure, volume, temperature) are property relations. An equation of state which would express the density in terms of pressure and temperature needs to be close to Equation (1). Several different equations of state, including SRK, PR, BWRS, AGA-8, GERG 88, and GERG 2004, are applied in the industry. The sensitivity of the selection of the equation of state for pipeline gas flow models was investigated by Chaczykowski [32]. In this paper, the BWRS equation of state [20,33] is used, and the BWRS equation is formulated as

$$
\begin{aligned}
p= & \rho R T+\left(B_{0} R T-A_{0}-\frac{C_{0}}{T^{2}}+\frac{D_{0}}{T^{3}}-\frac{E_{0}}{T^{4}}\right) \rho^{2}+\left(b R T-a-\frac{d}{T}\right) \rho^{3} \\
& +\alpha\left(a+\frac{d}{T}\right) \rho^{6}+\frac{c \rho^{3}}{T^{2}}\left(1+\gamma \rho^{2}\right) \exp \left(-\gamma \rho^{2}\right)
\end{aligned}
$$

In total, it contains 11 coefficients. Values and mixing rules can be found in the literature [20,33].

\subsubsection{Non-Pipeline Model}

The main non-pipeline components in the natural gas pipeline network are compressor and valve components. Their mathematical models can be found in much literature [12,23]. In this paper, the mathematical models of compressors and valves are presented as Equations (3) to (5) and Equations (6) to (7), respectively.

Compressors model:

$$
\begin{gathered}
m_{\text {in }}-m_{\text {out }}=0, \\
p_{\text {out }}-\varepsilon p_{\text {in }}=0, \\
T_{\text {out }}-T_{\text {in }} \varepsilon^{\frac{n-1}{n}}=0,
\end{gathered}
$$

where, $\varepsilon=a+b Q_{\text {in }}+c Q_{\text {in }}^{2}$.

Valves model:

$$
\begin{gathered}
m_{\text {in }}-m_{\text {out }}=0, \\
m_{\text {in }}-C_{v} \rho_{\text {in }} \sqrt{\frac{p_{\text {in }}^{2}-p_{\text {out }}^{2}}{Z \Delta T_{\text {in }}}}=0, \\
T_{\text {out }}-T_{\text {in }}+\left(p_{\text {out }}-p_{\text {in }}\right)\left\{\frac{1}{\mathcal{c}_{p}}\left[\frac{T}{\rho^{2}} \frac{(\partial p / \partial T)_{\rho}}{(\partial p / \partial \rho)_{T}}-\frac{1}{\rho}\right]\right\}_{\text {out }}=0,
\end{gathered}
$$

\subsubsection{Multi-Component Interconnection Node Model}

In each multi-component interconnection node, the laws of mass conservation, the equality of pressure, and the equality of gas temperature must be observed $[12,20,26,28,31]$. The corresponding mathematical models are represented by Equations (9) to (10). 
Mass conservation:

$$
\sum m_{\text {in }}=\sum m_{\text {out }}
$$

Equality of pressure:

$$
p_{\text {in }, 1}=p_{\text {in }, 2} \cdots=p_{\text {out }, 1}=p_{\text {out }, 2} \cdots,
$$

Equality of gas temperature outflow from the node:

$$
T_{\text {out }, 1}=T_{\text {out }, 2} \cdots=\sum\left|c_{p} m T\right|_{\text {in }} / \sum\left|c_{p} m\right|_{\text {out }},
$$

\subsubsection{Boundary Conditions}

The boundary conditions give the value of pressure, flow rate, and temperature of the external components $[12,26,28,31]$. The external components of the gas pipeline network include the supply and the demand. The supply is the component where gas is injected into the network, and the demand is the component where gas is extracted from the network. The boundary conditions are represented by Equations (12) to (14).

Pressure:

$$
p=p(t)
$$

Flow rate:

$$
m=m(t)
$$

Temperature:

$$
T=T(t),
$$

\subsection{Discretization}

The pipeline model, that is Equation (1), is a nonlinear partial differential equation. It can be linearized about the previous time step based on the Taylor expansion, as shown below [12,17,18,23,24,26]:

$$
\frac{\partial \mathbf{U}}{\partial t}+\overline{\mathbf{B}} \cdot \frac{\partial \mathbf{U}}{\partial x}+\overline{\mathbf{G}} \cdot(\mathbf{U}-\overline{\mathbf{U}})=\overline{\mathbf{F}}+\overline{\mathbf{S}} \cdot(\mathbf{U}-\overline{\mathbf{U}}),
$$

where, $[\mathbf{G}]_{i, j}=\sum_{l=1}^{n}\left(\frac{\partial B}{\partial u_{j}}\right)_{i, l} \frac{\partial u_{l}}{\partial x},[\mathbf{S}]_{i, j}=\frac{\partial F_{i}}{\partial u_{j}}$. The detailed expressions of $\mathbf{G}$ and $\mathbf{S}$ can be found in appendix A. It should be noted that the bar " above the matrixes $\mathbf{B}, \mathbf{G}, \mathbf{F}, \mathbf{S}$, and $\mathbf{U}$ represents the previous time step, so $\overline{\mathbf{B}}, \overline{\mathbf{G}}, \overline{\mathbf{F}}, \overline{\mathbf{S}}$, and $\overline{\mathbf{U}}$ are calculated by the results of the previous time step.

The pipeline is divided into $N$ sections, and thus, there are $N+1$ grid points. The $i$ th section is the section between the $i$ th point and the $(i+1)$ th point. Figure 1 is the schematic diagram of the pipeline grid.

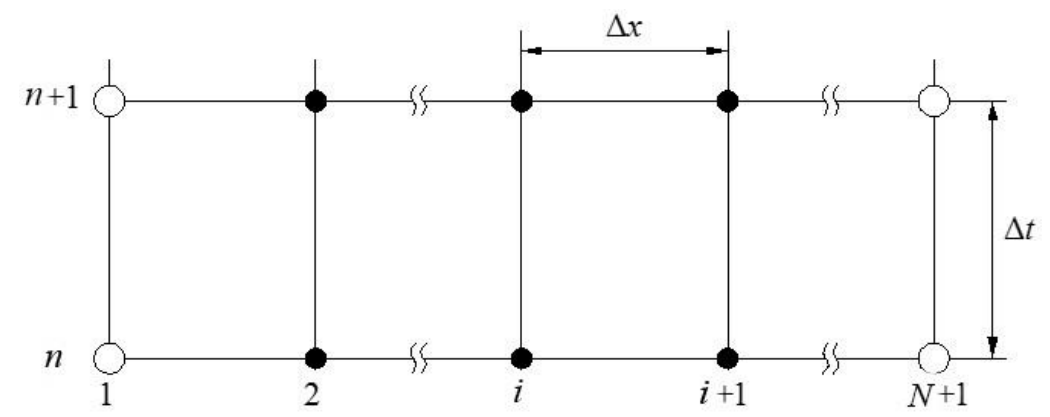

Figure 1. Grids of the pipeline 
The flow equations of Equation (15) are discretized by the central difference scheme in the $i$ th section in Figure 1, and the discretized flow equations are obtained as Equation (16) [26,28,31]:

$$
\mathbf{C E}_{i} \cdot \mathbf{U}_{i}^{n+1}+\mathbf{D W}_{i} \cdot \mathbf{U}_{i+1}^{n+1}=\mathbf{H}_{i}
$$

where, $\mathbf{C E}_{i}=\frac{1}{2 \Delta t} \mathbf{I}-\frac{1}{\Delta x} \overline{\mathbf{B}}+\frac{1}{2}(\overline{\mathbf{G}}-\overline{\mathbf{S}}) ; \mathbf{D W}_{i}=\frac{1}{2 \Delta t} \mathbf{I}+\frac{1}{\Delta x} \overline{\mathbf{B}}+\frac{1}{2}(\overline{\mathbf{G}}-\overline{\mathbf{S}}) ; \mathbf{H}_{i}=\overline{\mathbf{F}}+\left(\overline{\mathbf{G}}-\overline{\mathbf{S}}+\frac{1}{\Delta t}\right) \cdot \overline{\mathbf{U}}$.

The thermodynamic equation of Equation (15) is discretized by the upwind scheme at the ith point in Figure 1, and the discretized thermodynamic equation is obtained as Equation (17) [23,29,31]:

$$
U P_{i} \cdot T_{i-1}^{n+1}+C E_{i} \cdot T_{i}^{n+1}+D W_{i} \cdot T_{i+1}^{n+1}=H_{i}
$$

where, $U P_{i}=-\max \left(w_{i}^{n+1}, 0\right) \frac{1}{\Delta x} ; C E_{i}=\left(\frac{1}{\Delta t}-\bar{S}+\left|w_{i}^{n+1}\right| \frac{1}{\Delta x}\right) ; D W_{i}=-\max \left(-w_{i}^{n+1}, 0\right) \frac{1}{\Delta x}, H_{i}=F\left(T_{i}^{n}\right)+$ $\left(\frac{1}{\Delta t}-\bar{S}\right) T_{i}^{n}$.

Equations (3) to (17) are the flow and thermodynamic-coupled discretized equations of the natural gas pipeline network. These equations are split into two parts: the system of flow equations and the system of thermodynamic equations.

The system of flow equations consists of Equations (3) to (4), Equations (6) to (7), Equations (9) to (10), Equations (12) to (13), and equation (16). The system of thermodynamic equations consists of Equation (5), Equation (6), Equation (11), Equation (14), and Equation (17).

The decoupled solution strategy [23-25] is adopted to efficiently solve the flow and thermodynamic-coupled discretized equations. In the decoupled solution strategy, the discretized flow equations are firstly solved using the interpolated temperature, and the discretized thermodynamic equations are then solved using the solved flow variables, such as the pressure and flow rate. The decoupled solution strategy is shown in Figure 2.

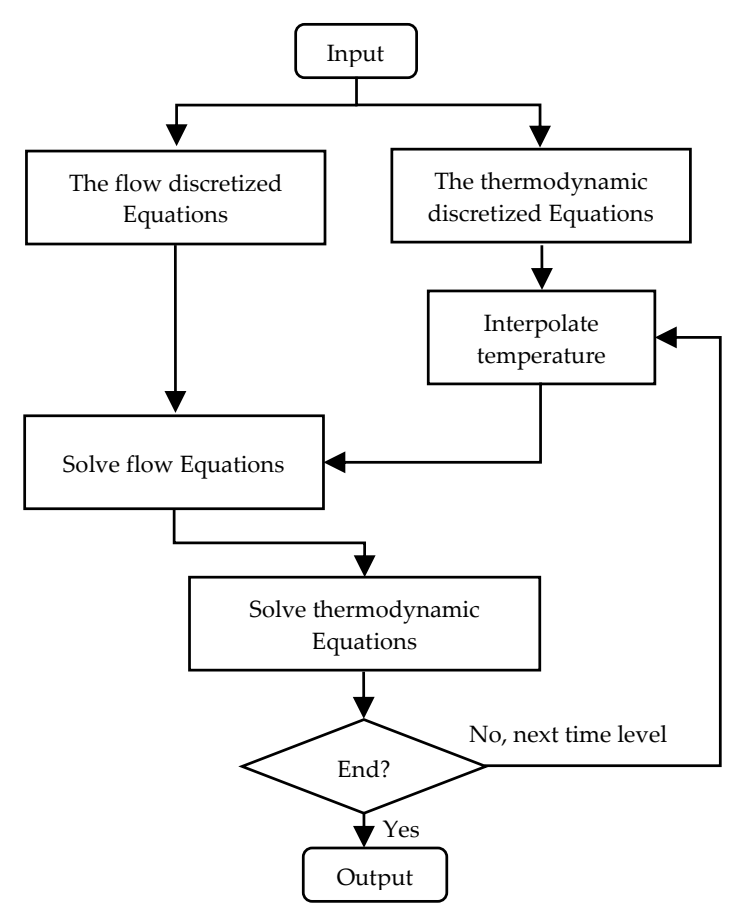

Figure 2. The decoupled strategy.

\section{Main Idea of the DIMENS Method}

In the DIMENS method, the network is firstly divided into several independent pipelines, and the pipelines are then efficiently solved one by one. Figure 3 shows an example, where a pipeline network is divided into four interconnection nodes and three pipelines. 


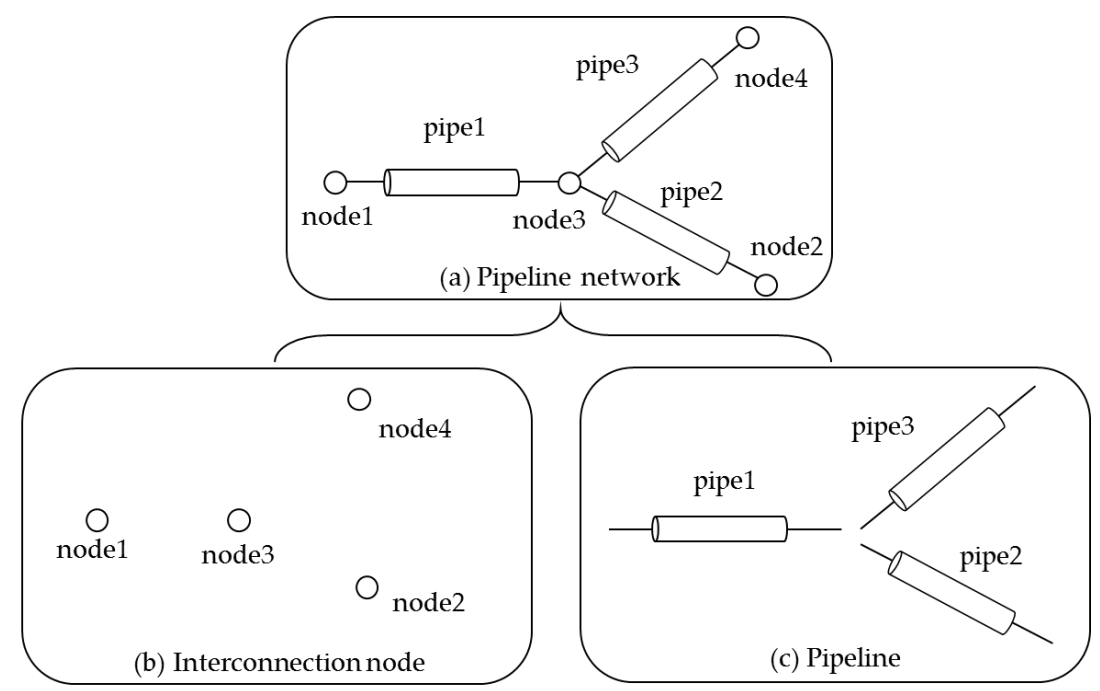

Figure 3. Divide-and-conquer approach for the natural gas pipeline network.

The key to decoupling the pipelines is solving the multi-pipeline interconnection nodes. However, the equation number of the multi-pipeline interconnection nodes is less than the number of the unknowns to be solved, so those equations of nodes cannot be solved directly.

Our previous work [28] is the first report on how to solve the above problem. The idea of solving the problem is that 'the supplemental equations for solving the multi-pipeline interconnection nodes are obtained from the discretized equations of the pipeline'. In other words, the discretized equations of pipelines are solved in advance to obtain supplemental equations, and the equations of multi-pipeline interconnection nodes are then solved with the supplemental equations. Figure 4 shows the procedure of the DIMENS method for the pipeline network.

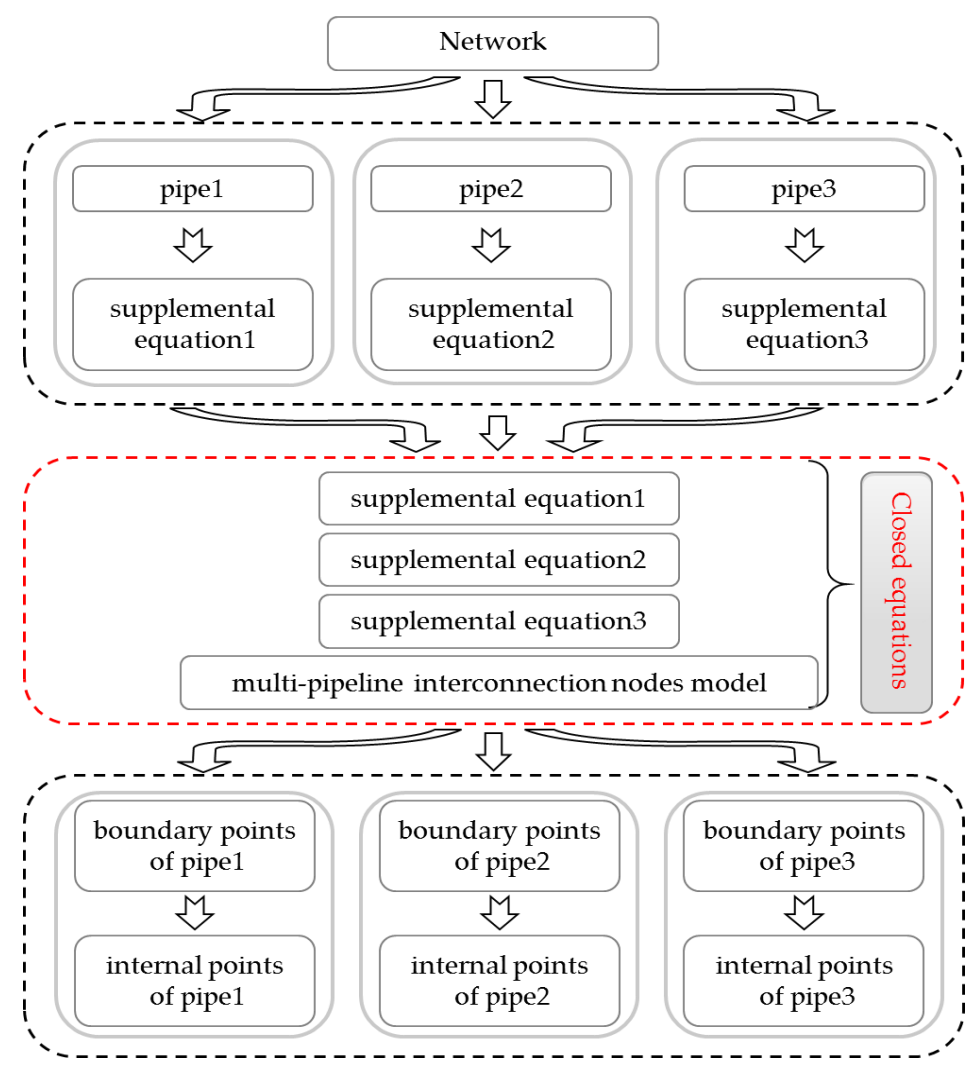

Figure 4. Procedure of the DIMENS method for the pipeline network. 
In our previous work [23], the DIMENS method was only implemented in flow simulation of the simple pipeline network, which is only composed of pipelines. In the following section, the DIMENS method is extended to the flow and thermodynamic-coupled simulation of the complex pipeline network, which includes pipelines and non-pipeline components. Based on the flow and thermodynamic-decoupled solution strategy [23-25], the DIMENS method is implemented in the flow simulation and thermodynamic simulation, respectively.

\section{Extended DIMENS for Complex Pipeline Network}

\subsection{Implementation of the Flow Simulation of the Complex Pipeline Network}

\subsubsection{Analysis of Flow Simulation}

In our previous work [28], the process of solving flow Equations by the DIMNES method has been discussed in detail as step1, step2, and step3. However, the pipeline network does not involve non-pipeline components, such as compressor and valve components. In this paper, the non-pipeline components are appropriately solved as multi-component interconnection nodes in step 2 of the DIMENS method. The procedures of the DIMENS method in flow simulation are given below.

\subsubsection{Procedures of the DIMENS Method in Flow Simulation}

Step 1: Pre-solve pipeline

By taking the pressure of the starting point $p_{1}$ and the mass flow rate of the terminal point $m_{N+1}$ as free variables, Equation (16) can be solved by the block three diagonal matrix algorithm (BTDMA). It's general solution can be written as Equation (18). The detailed solution process can be viewed in previous literature [28]:

$$
\left[p_{1}, m_{1}, \cdots, p_{N+1}, m_{N+1}\right]^{T}=p_{1} \boldsymbol{\alpha}+m_{N+1} \boldsymbol{\beta}+\boldsymbol{\gamma},
$$

Equations (19) and (20) are the supplemental equations used to solve the multi-component interconnection nodes. These equations are the key to solving the multi-component interconnection nodes.

$$
\begin{gathered}
m_{1}=\alpha_{1}^{m} p_{1}+\beta_{1}^{m} m_{N+1}+\gamma_{1}^{m}, \\
p_{N+1}=\alpha_{N+1}^{p} p_{1}+\beta_{N+1}^{p} m_{N+1}+\gamma_{N+1^{\prime}}^{p}
\end{gathered}
$$

Step 2: Solve the multi-component interconnection node

With the supplemental equations obtained from step 1, the equations of multi-component interconnection nodes are closed and can be solved. The closed equations are composed of four parts: (1) the flow equations of the non-pipeline model, Equations (3) to (4) and Equations (6) to (7); (2) the flow equations of the multi-component interconnection node model, Equations (9) to (10); (3) the flow equations of boundary conditions, Equations (12) to (13); and (4) the supplemental equations obtained from the first step, Equations (19) to (20). The unknown variables of the closed equations are the mass flow rates and pressures of the starting and terminal points of all components. The preconditioned conjugate gradient (CG) algorithm proposed in our previous work [28] is employed to solve the closed equations. For better understanding how the equations of multi-component interconnection nodes are solved, a simple pipeline network is given as the example in Appendix B.

Step 3: Solve the internal points of the pipeline

The values of the free variables $\left(p_{1}\right.$ and $\left.m_{N+1}\right)$ solved in step 2 are substituted into Equations (19) to (20). The flow parameters of the internal grid points of the pipelines can be easily obtained to complete the flow simulation of the whole pipeline network. Through the above three steps, the flow simulation of the complex pipeline network can be solved by the DIMENS method. 


\subsection{Implementation of the Thermodynamic Simulation of the Complex Pipeline Network}

\subsubsection{Analysis of Thermodynamic Simulation}

It is generally known that the downstream propagation of natural gas temperature is dominated by gas flow in the pipeline, and only upstream gas affects downstream gas. Therefore, only the temperature of the pipeline inlet can be chosen as the free variable for the DIMENS method to solve the thermodynamic equations. However, the direction of gas flow in the pipeline can unpredictably change during the transience. This means that gas could flow into the pipeline either from the starting or terminal point of the pipeline. Therefore, the flow states of gas in the pipeline should be analyzed to choose the free thermodynamic variables for the DIMENS method.

During the transience of the natural gas flow in the pipeline, there are four kinds of flow states: (1) natural gas flows out of the pipeline from both the starting and terminal points; (2) natural gas flows into the pipeline from the starting point and flows out of the pipeline from the terminal point; (3) natural gas flows into the pipeline from the terminal point and flows out of the pipeline from the starting point; and (4) natural gas flows into the pipeline from both the starting and terminal points. According to the corresponding gas flow state, pipelines can be classified as four types. The flow states and types of the pipeline are shown in Table 2.

Table 2. The flow states and the solution conditions of the pipeline.

First type pipeline

For the first type of pipeline, the thermodynamic-discretized equation of the pipeline model, Equation (17), is a closed system. This means that there is no free variable, and the first type of pipeline can be solved directly. For the second type of pipeline, Equation (17) is not a closed system until the temperature of the starting point is known. That is to say, the temperature of the starting point should be chosen as the free variable. Similar to the second type of pipeline, the free variable of the third type of pipeline is the temperature of the terminal point. For the fourth type of pipeline, until the temperatures of the starting and terminal points are both known, the temperature of the internal grid point in the pipeline can be obtained by solving Equation (17). In other words, the fourth type of pipeline should be solved after the other three pipeline types. The solution requirements of these four types of pipelines are also shown in Table 2.

\subsubsection{Procedures of the DIMENS Method in Thermodynamic Simulation}

According to the above analysis of the pipeline flow state, the DIMENS method is extended to the thermodynamic simulation of the complex pipeline network, and the detailed procedures are presented as follows.

Step 1: Classify pipelines

Pipelines are classified as four types according to flow state, shown in Table 2.

Step 2: Solve the first type pipeline 
The objective of this step is to obtain the thermodynamic parameters of the first type of pipeline. For the first type of pipeline, Equation (17) is closed, which can be rewritten as Equation (21). Then, Equation (21) is solved efficiently by the three diagonal matrix algorithm (TDMA) [34], and the temperature values of all points on the first type pipeline are obtained:

$$
\left[\begin{array}{cccc}
C E_{1} & D W_{1} & & \\
U P_{2} & C E_{2} & D W_{2} & \\
& \ddots & \ddots & \\
& U P_{N} & C E_{N} & D W_{N} \\
& & U P_{N+1} & C E_{N+1}
\end{array}\right]\left[\begin{array}{c}
T_{1} \\
T_{2} \\
\vdots \\
T_{N} \\
T_{N+1}
\end{array}\right]=\left[\begin{array}{c}
H_{1} \\
H_{2} \\
\vdots \\
H_{N} \\
H_{N+1}
\end{array}\right]
$$

Step 3: Pre-solve the second and third type pipelines

By taking the temperature of starting point $T_{1}$ of the second type of pipeline as the free variable, Equation (17) can be rewritten as Equation (22), and its general solution is Equation (23). Similar to the second type of pipeline, the temperature of terminal point $T_{N+1}$ of the third type of pipeline is the free variable, and the corresponding discretized Equation and general solution are Equation (24) and Equation (25), respectively. In addition, the TDMA algorithm [34] is adopted too.

Thermodynamic-discretized equations of the second type of pipeline:

$$
\left[\begin{array}{cccc}
1 & 0 & & \\
U P_{2} & C E_{2} & D W_{2} & \\
& \ddots & \ddots & \\
& U P_{N} & C E_{N} & D W_{N} \\
& & U P_{N+1} & C E_{N+1}
\end{array}\right]\left[\begin{array}{cc}
\alpha_{1} & \gamma_{1} \\
\alpha_{2} & \gamma_{2} \\
\vdots & \vdots \\
\alpha_{N} & \gamma_{N} \\
\alpha_{N+1} & \gamma_{N+1}
\end{array}\right]=\left[\begin{array}{cc}
1 & 0 \\
0 & H_{2} \\
\vdots & \vdots \\
0 & H_{N} \\
0 & H_{N+1}
\end{array}\right]
$$

General solution of the second type of pipeline:

$$
\left[\begin{array}{lllll}
T_{1} & T_{2} & \cdots & T_{N} & T_{N+1}
\end{array}\right]^{T}=T_{1} \boldsymbol{\alpha}+\boldsymbol{\gamma},
$$

Thermodynamic-discretized equations of the third type of pipeline:

$$
\left[\begin{array}{cccc}
C E_{1} & D W_{1} & & \\
U P_{2} & C E_{2} & D W_{2} & \\
& \ddots & \ddots & \\
& U P_{N} & C E_{N} & D W_{N} \\
& & 0 & 1
\end{array}\right]\left[\begin{array}{cc}
\alpha_{1} & \gamma_{1} \\
\alpha_{2} & \gamma_{2} \\
\vdots & \vdots \\
\alpha_{N} & \gamma_{N} \\
\alpha_{N+1} & \gamma_{N+1}
\end{array}\right]=\left[\begin{array}{cc}
0 & H_{1} \\
0 & H_{2} \\
\vdots & \vdots \\
0 & H_{N} \\
1 & 0
\end{array}\right]
$$

General solution of the third type of pipeline:

$$
\left[\begin{array}{lllll}
T_{1} & T_{2} & \cdots & T_{N} & T_{N+1}
\end{array}\right]^{T}=T_{N+1} \boldsymbol{\alpha}+\boldsymbol{\gamma},
$$

Equation (26) and Equation (27) are the supplemental equations used to solve the multi-component interconnection nodes. These equations are the key to solving the multi-component interconnection nodes.

$$
\begin{gathered}
T_{N+1}=T_{1} \alpha_{N+1}+\gamma_{N+1}, \\
T_{1}=T_{N+1} \alpha_{1}+\gamma_{1},
\end{gathered}
$$

Step 4: Solve the multi-components interconnection node

In the DIMENS method, the fourth step of thermodynamic equations is similar to the second step of the flow equations. The thermodynamic parameters of multi-components interconnection 
nodes are solved via the closed equations of the starting and terminal points of all components. The complete system of closed equations is comprised of five parts: (1) thermodynamic equations of the non-pipeline model, Equation (5) and Equation (6); (2) thermodynamic equation of the multi-components interconnection node model, Equation (11); (3) thermodynamic equation of the boundary condition, Equation (14); (4) the temperature values of the starting and terminal points of the first type of pipeline that are solved in step 2; and (5) the supplemental equations obtained in step 3, Equation (26) and Equation (27). The unknown variables of these closed equations are the temperatures of the starting and terminal points of all components. The preconditioned CG algorithm that is proposed in the paper [34] is adopted to solve the closed equations. For better understanding how the equations of multi-component interconnection nodes are solved in thermodynamic equations, a simple pipeline network is given as the example in Appendix C.

Step 5: Solve the internal points of the second and third pipelines

The fifth step of thermodynamic equations is similar to the third step of the flow equations. The values of the free variables solved in step 4 are substituted into Equation (23) and Equation (25), and the temperature value of the internal grid points of the first and second types of pipelines can be easily obtained.

Step 6: Solve the fourth type pipeline

After step 5, the temperatures of the starting and terminal points of all pipelines are known. Then, Equation (17) of the fourth type of pipeline is rewritten as Equation (28), and is efficiently solved by the TDMA algorithm [34].

$$
\left[\begin{array}{cccc}
1 & 0 & & \\
U P_{2} & C E_{2} & D W_{2} & \\
& \ddots & \ddots & \\
& U P_{N} & C E_{N} & D W_{N} \\
& & 0 & 1
\end{array}\right]\left[\begin{array}{c}
T_{1} \\
T_{2} \\
\vdots \\
T_{N} \\
T_{N+1}
\end{array}\right]=\left[\begin{array}{c}
T_{1}^{s} \\
H(2) \\
\vdots \\
H(N) \\
T_{N+1}^{s}
\end{array}\right],
$$

Through the above six steps, thermodynamic simulation of the complex pipeline network can be solved by the DIMENS method.

\section{Results and Analysis}

An example of the complex pipeline is designed to validate the DIMENS method here. The calculation accuracy of the DIMENS method is investigated by comparing the numerical solution obtained by the DIMENS method with that of SPS. The computational speed of the DIMENS method is investigated by comparing the CPU time of the DIMENS method with that of SPS.

\subsection{Description of the Numerical Test}

The complex pipeline is comprised of 84 pipelines, four compressors, eight valves, and 51 externals, and the structure of the complex pipeline network is shown in Figure 5 and detailed data are given in Tables 3-6. All the pipelines are horizontal, with a roughness, thickness, and thermal conductivity of $0.02286 \mathrm{~mm}, 5 \mathrm{~mm}$, and $0.0127 \mathrm{~W} /(\mathrm{m} \cdot \mathrm{K})$, respectively. 


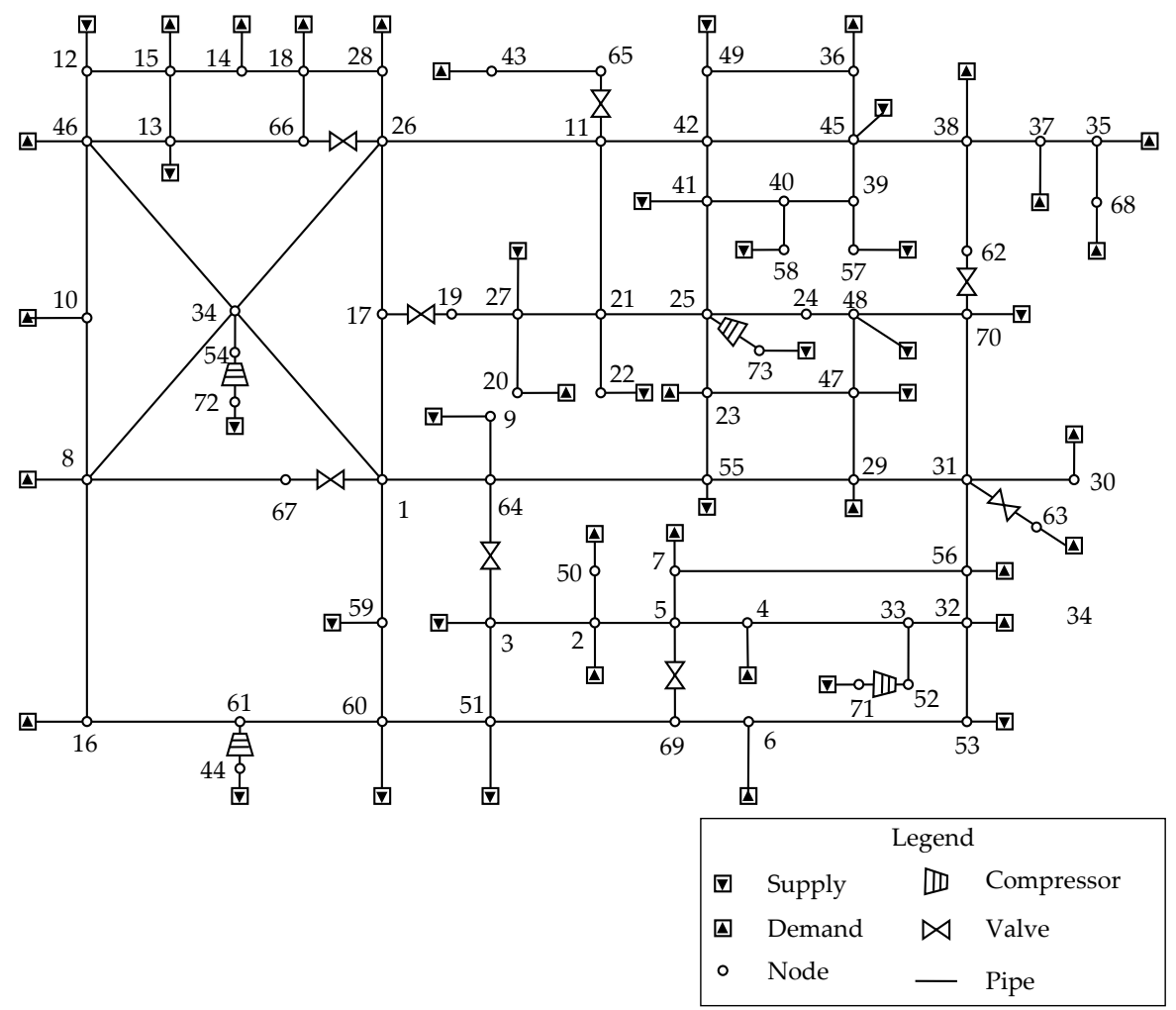

Figure 5. The schematic diagram of the topology structure of the complex pipeline network.

Table 3. Pipeline data.

\begin{tabular}{|c|c|c|c|c|c|c|c|c|c|c|c|c|c|c|}
\hline Pipeline & 1 & 2 & 3 & 4 & 5 & 6 & 7 & 8 & 9 & 10 & 11 & 12 & 13 & 14 \\
\hline Starting node & 64 & 3 & 5 & 51 & 5 & 69 & 5 & 67 & 8 & 9 & 46 & 15 & 13 & 66 \\
\hline Ending node & 1 & 2 & 2 & 3 & 4 & 6 & 7 & 8 & 10 & 64 & 10 & 12 & 46 & 13 \\
\hline Length (km) & 52 & 78 & 66 & 40 & 23 & 22 & 39 & 58 & 50 & 60 & 35 & 59 & 57 & 56 \\
\hline Diameter (mm) & 325 & 325 & 325 & 219 & 325 & 219 & 325 & 273 & 457 & 168 & 273 & 355 & 273 & 219 \\
\hline Pipeline & 15 & 16 & 17 & 18 & 19 & 20 & 21 & 22 & 23 & 24 & 25 & 26 & 27 & 28 \\
\hline Starting node & 13 & 14 & 1 & 17 & 66 & 18 & 19 & 27 & 27 & 22 & 25 & 25 & 24 & 26 \\
\hline Ending node & 15 & 15 & 17 & 26 & 18 & 14 & 27 & 20 & 21 & 21 & 21 & 23 & 25 & 28 \\
\hline Length (km) & 60 & 63 & 20 & 46 & 36 & 75 & 41 & 33 & 33 & 42 & 78 & 83 & 45 & 135 \\
\hline Diameter (mm) & 273 & 168 & 457 & 457 & 273 & 325 & 219 & 219 & 168 & 168 & 355 & 355 & 355 & 457 \\
\hline Pipeline & 29 & 30 & 31 & 32 & 33 & 34 & 35 & 36 & 37 & 38 & 39 & 40 & 41 & 42 \\
\hline Starting node & 55 & 31 & 31 & 32 & 32 & 53 & 52 & 8 & 37 & 38 & 45 & 45 & 39 & 40 \\
\hline Ending node & 64 & 29 & 30 & 56 & 33 & 6 & 33 & 61 & 35 & 37 & 38 & 39 & 40 & 41 \\
\hline Length (km) & 92 & 46 & 74 & 50 & 40 & 77 & 25 & 85 & 63 & 85 & 69 & 44 & 76 & 67 \\
\hline Diameter (mm) & 457 & 457 & 355 & 508 & 508 & 219 & 508 & 426 & 426 & 426 & 426 & 426 & 219 & 355 \\
\hline Pipeline & 43 & 44 & 45 & 46 & 47 & 48 & 49 & 50 & 51 & 52 & 53 & 54 & 55 & 56 \\
\hline Starting node & 11 & 65 & 56 & 47 & 11 & 26 & 2 & 41 & 45 & 12 & 53 & 54 & 29 & 31 \\
\hline Ending node & 26 & 43 & 7 & 48 & 21 & 34 & 50 & 42 & 42 & 46 & 32 & 34 & 55 & 56 \\
\hline Length (km) & 115 & 46 & 74 & 53 & 63 & 86 & 63 & 72 & 142 & 93 & 80 & 53 & 102 & 50 \\
\hline Diameter (mm) & 426 & 273 & 273 & 219 & 219 & 355 & 219 & 426 & 426 & 325 & 325 & 508 & 426 & 426 \\
\hline Pipeline & 57 & 58 & 59 & 60 & 61 & 62 & 63 & 64 & 65 & 66 & 67 & 68 & 69 & 70 \\
\hline Start & 57 & 58 & 59 & 60 & 61 & 35 & 69 & 1 & 31 & 8 & 16 & 61 & 60 & 38 \\
\hline Ending node & 39 & 40 & 1 & 59 & 1 & 68 & 51 & 34 & 70 & 16 & 61 & 60 & 51 & 62 \\
\hline Length (km) & 30 & 68 & 43 & 56 & 52 & 36 & 45 & 52 & 52 & 80 & 84 & 101 & 95 & 33 \\
\hline Diameter (mm) & 168 & 325 & 273 & 273 & 325 & 355 & 273 & 355 & 355 & 273 & 273 & 426 & 426 & 325 \\
\hline Pipeline & 71 & 72 & 73 & 74 & 75 & 76 & 77 & 78 & 79 & 80 & 81 & 82 & 83 & 84 \\
\hline Starting node & 41 & 23 & 33 & 18 & 49 & 45 & 49 & 8 & 46 & 11 & 24 & 48 & 47 & 23 \\
\hline Ending node & 25 & 55 & 4 & 28 & 42 & 36 & 36 & 34 & 34 & 42 & 48 & 70 & 29 & 47 \\
\hline Length $(\mathbf{k m})$ & 68 & 42 & 42 & 68 & 52 & 50 & 43 & 52 & 59 & 66 & 49 & 63 & 76 & 53 \\
\hline Diameter (mm) & 355 & 273 & 457 & 273 & 273 & 273 & 219 & 355 & 325 & 355 & 355 & 325 & 325 & 219 \\
\hline
\end{tabular}

Note: Diameter in Table 3 is outside diameter. 
Table 4. Compressor data.

\begin{tabular}{ccccc}
\hline Compressor & 1 & 2 & 3 & 4 \\
\hline Starting point & 71 & 72 & 73 & 74 \\
Ending point & 52 & 54 & 25 & 61 \\
\hline
\end{tabular}

Table 5. Valve data.

\begin{tabular}{ccccccccc}
\hline Valve & 1 & 2 & 3 & 4 & 5 & 6 & 7 & 8 \\
\hline Starting point & 70 & 31 & 17 & 64 & 11 & 1 & 26 & 5 \\
Ending point & 62 & 63 & 19 & 3 & 65 & 67 & 66 & 69 \\
\hline
\end{tabular}

Table 6. External data.

\begin{tabular}{cccccccccccccc}
\hline Externals & 1 & 2 & 3 & 4 & 5 & 6 & 7 & 8 & 9 & 10 & 11 & 12 & 13 \\
\hline Adjacent node & 37 & 53 & 45 & 47 & 48 & 56 & 51 & 3 & 49 & 71 & 70 & 13 & 12 \\
\hline Externals & 14 & 15 & 16 & 17 & 18 & 19 & 20 & 21 & 22 & 23 & 24 & 25 & 26 \\
\hline Adjacent node & 72 & 55 & 22 & 58 & 9 & 57 & 73 & 41 & 59 & 60 & 27 & 44 & 6 \\
\hline Externals & 27 & 28 & 29 & 30 & 31 & 32 & 33 & 34 & 35 & 36 & 37 & 38 & 39 \\
\hline Adjacent node & 7 & 8 & 23 & 46 & 28 & 20 & 50 & 4 & 16 & 29 & 38 & 43 & 30 \\
\hline Externals & 40 & 41 & 42 & 43 & 44 & 45 & 46 & 47 & 48 & 49 & & & \\
\hline Adjacent node & 2 & 36 & 63 & 10 & 15 & 18 & 14 & 35 & 68 & 32 & & & \\
\hline
\end{tabular}

The components of natural gas are listed in Table 7. The standard pressure and temperature are $101.325 \mathrm{kPa}$ and $20^{\circ} \mathrm{C}$, respectively. The Colebrook Equation is used as the friction Equation [35]. During simulation, the ambient temperature is maintained at $15^{\circ} \mathrm{C}$.

Table 7. Main components of natural gas.

\begin{tabular}{cccccc}
\hline Component & $\mathrm{CH}_{4}$ & $\mathrm{C}_{2} \mathrm{H}_{4}$ & $\mathrm{C}_{3} \mathrm{H}_{8}$ & $\mathrm{~N}_{2}$ & $\mathrm{CO}_{2}$ \\
\hline Volume fraction (\%) & 97.07 & 0.17 & 0.02 & 0.71 & 2.03 \\
\hline
\end{tabular}

The initial pressure is $2.8 \mathrm{MPa}$, the initial volume flow rate is zero, and the initial temperature is $15^{\circ} \mathrm{C}$. After $t=0 \mathrm{~h}$, the pressure of some externals is maintained, the volume flow rate of some externals increases suddenly, the compressors are started, and the valves are opened. During the simulation, the volume flow rate of some externals is constant, while the volume flow rate of other externals and the valve opening $(F R)$ of valves are changed, shown as Tables 8-10, respectively. It is assumed that: (1) all the compressors have the same performance curve as shown in Figure 6, the rated speed of the compressor is $6000 \mathrm{RPM}$, and the start time is 3 minutes; (2) all the valves are same, the flow coefficient of the valve in this paper is calculated by the Equation $C_{\mathrm{v}}=200-200 F R$, and the travel time of the valve is one minute.

Table 8. Constant boundary conditions - pressure of the external.

\begin{tabular}{ccccccc}
\hline Externals & 10 & 14 & 20 & 25 & 46 & 48 \\
\hline Pressure (MPa) & 2.8 & 2.8 & 2.8 & 2.8 & 2.5 & 2.5 \\
\hline
\end{tabular}


Table 9. Constant boundary conditions flow rate of the external (flow in).

\begin{tabular}{ccccccccccc}
\hline Externals & 1 & 2 & 3 & 5 & 6 & 7 & 8 & 9 & 11 & 12 \\
\hline $\begin{array}{c}\text { Volume flow rate } \\
\left(\mathbf{1 0}^{\mathbf{4}} \mathbf{~ N m}^{\mathbf{3}} / \mathbf{d}\right)\end{array}$ & -9 & 85 & 25 & 26 & -100 & 44 & 15 & 36 & 55 & 68 \\
\hline Externals & 15 & 16 & 17 & 18 & 19 & 21 & 22 & 23 & 24 & 27 \\
\hline $\begin{array}{c}\text { Volume flow rate } \\
\left(\mathbf{( 1 0}^{4} \mathbf{~ N m}^{3} / \mathbf{d}\right)\end{array}$ & 25 & 15 & 18 & 14 & 2 & 14 & 21 & 56 & 12 & -34 \\
\hline$\quad$ Externals & 28 & 29 & 30 & 31 & 32 & 33 & 35 & 36 & 37 & 38 \\
\hline $\begin{array}{c}\text { Volume flow rate } \\
\left(\mathbf{1 0 ^ { 4 }} \mathbf{~ N m}^{3} / \mathbf{d}\right)\end{array}$ & -142 & -13 & -150 & -45 & -13.7 & -14 & -35 & -22 & -13 & -44 \\
\hline$\quad$ Externals & 39 & 40 & 41 & 42 & 43 & 44 & 45 & 47 & 49 & \\
\hline $\begin{array}{c}\text { Volume flow rate } \\
\left(\mathbf{1 0} \mathbf{~ N m}^{3} / \mathbf{d}\right)\end{array}$ & -21 & -25 & -50 & -16 & -56 & -69.4 & -63 & -66 & -85 & \\
\hline
\end{tabular}

Table 10. Changed boundary conditions.

\begin{tabular}{|c|c|c|c|c|c|}
\hline & External 4 & External 13 & External 26 & External 34 & \multirow{2}{*}{$\begin{array}{c}\text { Valves 1-8 } \\
\text { FR }\end{array}$} \\
\hline & \multicolumn{4}{|c|}{ Volume flow rate $\left(10^{4} \mathrm{Nm}^{3} / \mathrm{d}\right)$} & \\
\hline $0 \mathrm{~h} \leq t<5 \mathrm{~h}$ & 22 & 32 & -80 & -68 & 1.0 \\
\hline $5 \mathrm{~h} \leq t<10 \mathrm{~h}$ & 0 & 0 & 0 & 0 & 0.1 \\
\hline$t \geq 10 \mathrm{~h}$ & 22 & 32 & -80 & -68 & 1.0 \\
\hline
\end{tabular}

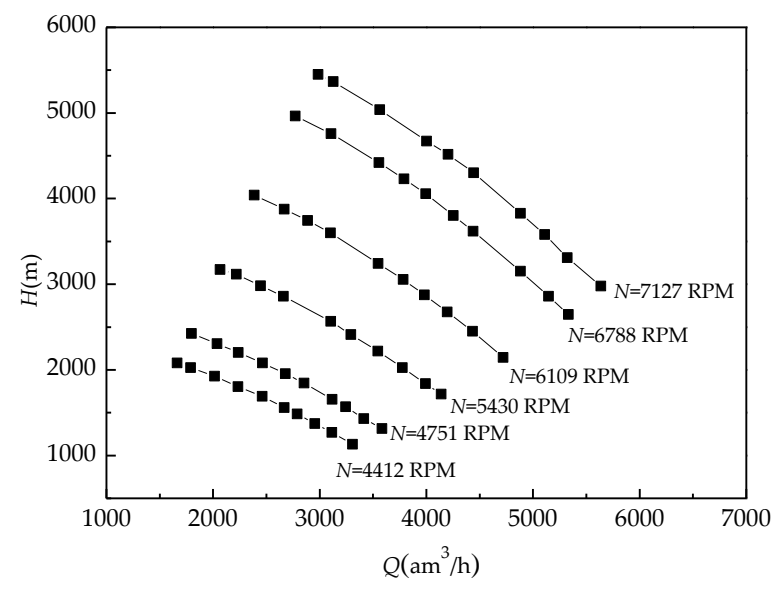

Figure 6. The performance curve of the compressor.

\subsection{Comparison of Numerical Accuracy}

The case of the complex pipeline network is simulated by the DIMENS and SPS, respectively. The time step and spatial step are $10 \mathrm{~s}$ and $0.5 \mathrm{~km}$, respectively. The pressure, volume flow rate, and temperature of the starting point of some pipelines during 0-10 $\mathrm{h}$ are shown in Figures 7-9. The pressure, volume flow rate, and temperature of the compressor 1\# are shown in Figure 10.

Figures 7-9 show that the numerical solutions of flow simulation, such as pressure and flow rate, obtained by the DIMENS method, are in good agreement with those obtained by SPS. The results of these comparisons are the same as in previous literature [28]. This can validate the DIMENS method for flow equations of the complex pipeline network. In addition, it is clearly seen from Figure 9 that the difference between the temperature obtained by the DIMENS method and that obtained by SPS is very small. The maximum deviation of temperature of the two methods is less than $1^{\circ} \mathrm{C}$, which can be found at the starting point of pipe 63 at $t=6 \mathrm{~h}$, as shown in Figure $9 \mathrm{~b}$. This is acceptable for practical engineering problems of natural gas pipeline transportation. That is to say, the DIMENS method is also accurate for the thermodynamic simulation. Additionally, the results of pressure, flow rate, and temperature of the compressor obtained by the DIMENS method are also in good agreement with 
those of SPS, as shown in Figure 10. This means that the complex start-up process of the compressor can be accurately simulated by the DIMENS method too. The above analysis shows that the DIMENS method has a high accuracy comparable to the accuracy of the SPS and can meet the requirements of practical engineering problems.

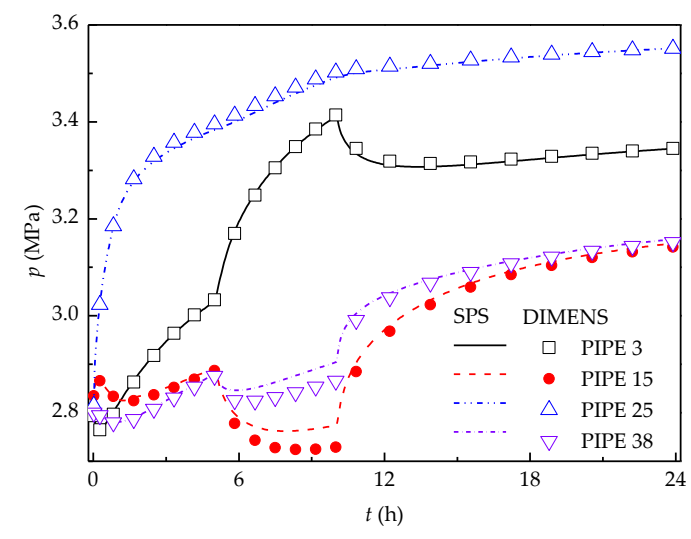

(a)

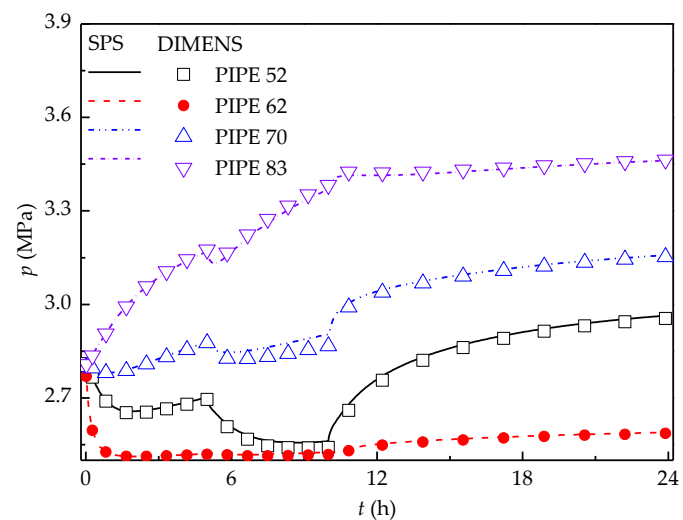

(b)

Figure 7. Pressure of the starting point of some pipelines: (a) Pipe 3, 15, 25, and 38; (b) Pipe 52, 62, 70, and 83 .

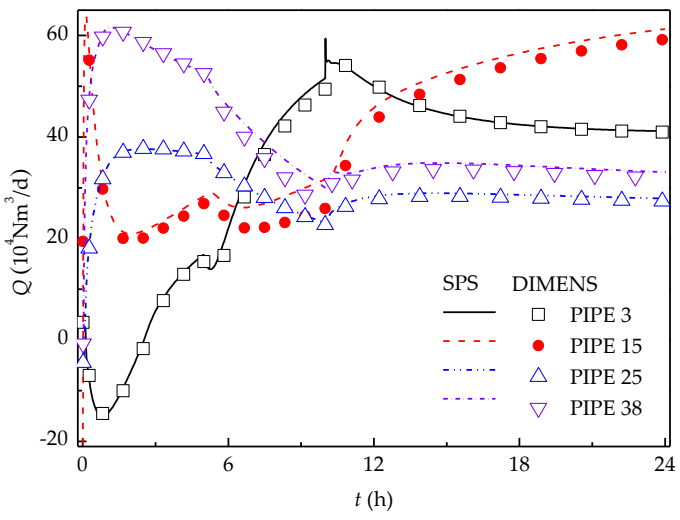

(a)

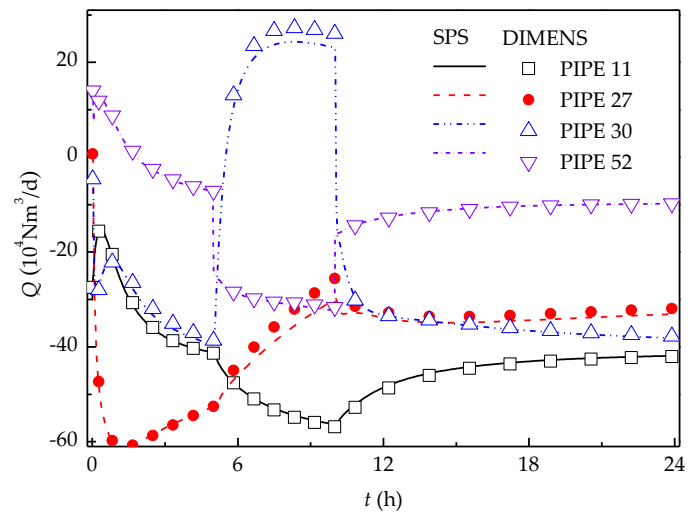

(b)

Figure 8. Volume flow rate of the starting point of some pipelines: (a) Pipe 1, 62, 72, and 81; (b) Pipe 11, 27,30 , and 52 .

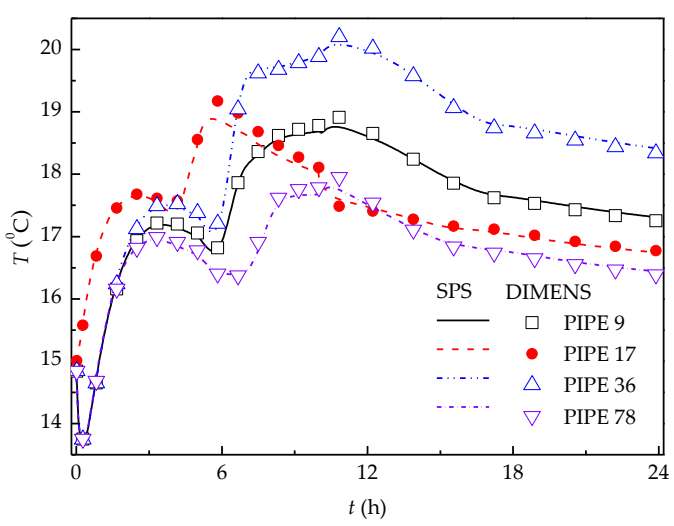

(a)

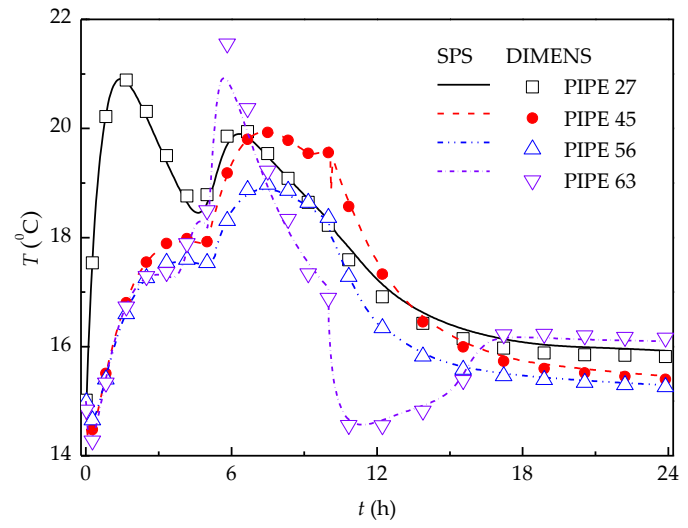

(b)

Figure 9. Temperature of the starting point of some pipelines: (a) Pipe 9, 17, 36, and 78; (b) Pipe 27, 45, 56 , and 63. 


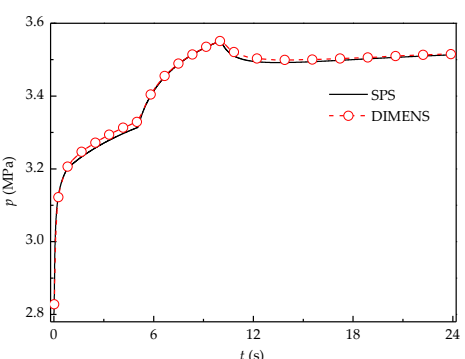

(a)

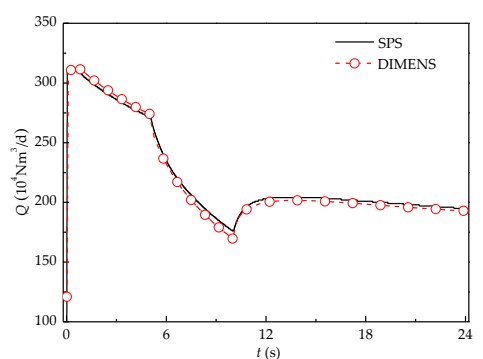

(b)

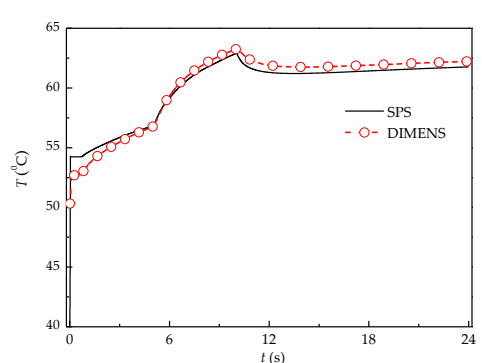

(c)

Figure 10. Pressure, flow rate, and temperature at the outlet of the 1\#compressor outlet: (a) Pressure; (b) flow rate; (c) temperature.

To further test the simulation accuracy of the DIMENS method, the results of the pipeline network at $t=24 \mathrm{~h}$ obtained by the DIMENS method and SPS are compared, as shown in Figures 11-13.

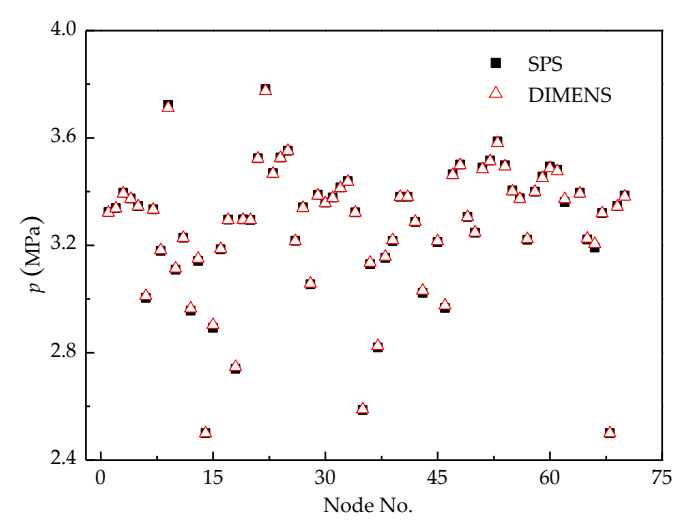

(a)

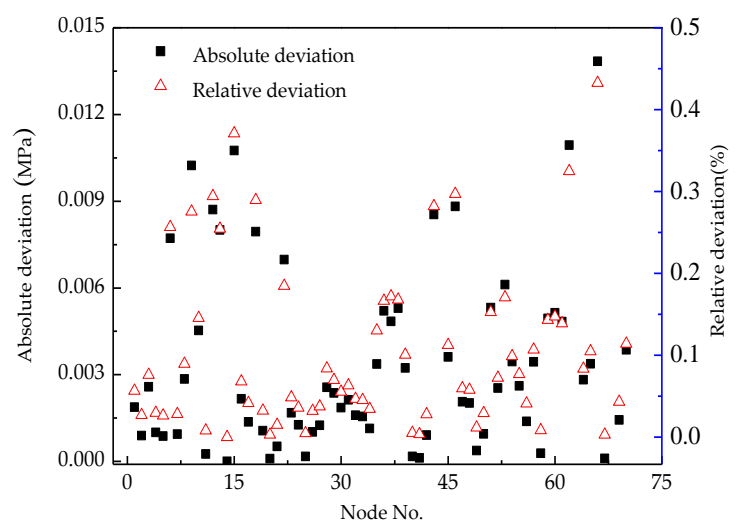

(b)

Figure 11. Comparison of pressure at the connection node: (a) Pressure value; (b) deviation.

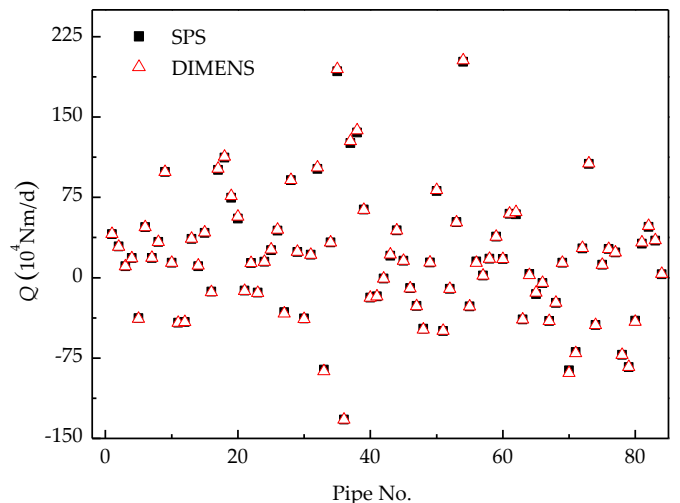

(a)

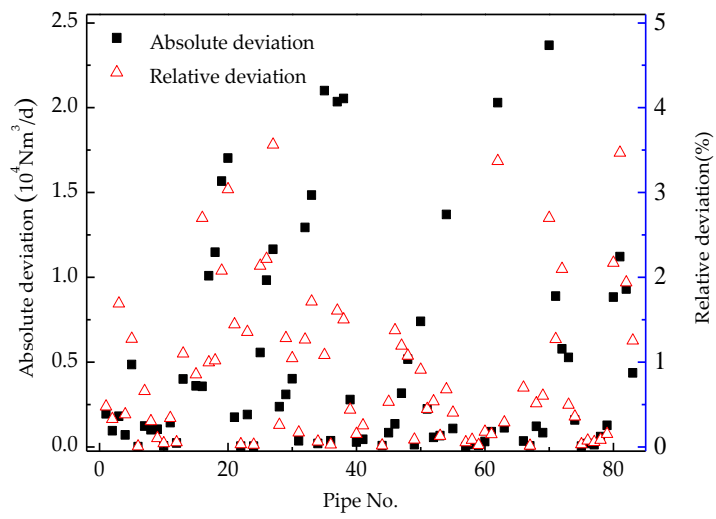

(b)

Figure 12. Comparison of flow rate of pipelines: (a) Flow rate value; (b) deviation.

From the comparison of the pressure of the connection nodes in Figure 11, it can be seen that the pressures obtained by the two methods are almost the same, and the maximum absolute and relative deviations of pressure are $0.015 \mathrm{MPa}$ and $0.45 \%$, respectively. From the comparison of the volume flow rate in Figure 12, the maximum absolute and relative deviations of the volume flow rate are $2.4 \times 10^{4} \mathrm{Nm}^{3} / \mathrm{h}$ and $3.5 \%$, respectively. What is more, the maximum absolute deviation of temperature is less than $0.5^{\circ} \mathrm{C}$, as shown in Figure 13. It can be summarized that the deviation between 
the numerical solution calculated by the DIMENS method and SPS is sufficiently small. These analyses again imply that the calculation accuracy of the DIMENS method is comparable to that of SPS.

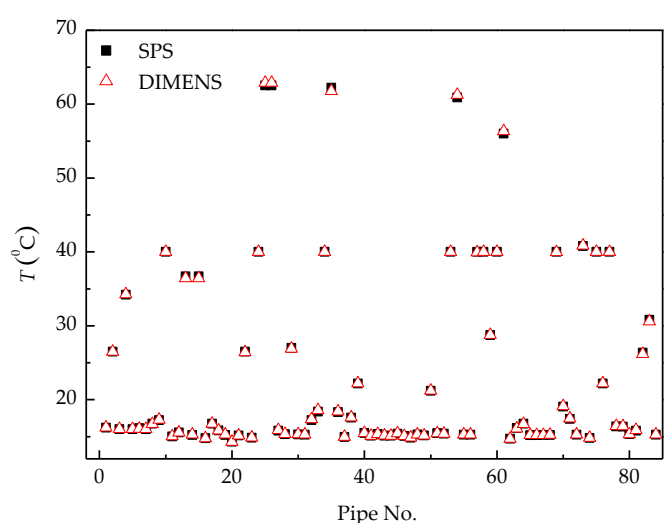

(a)

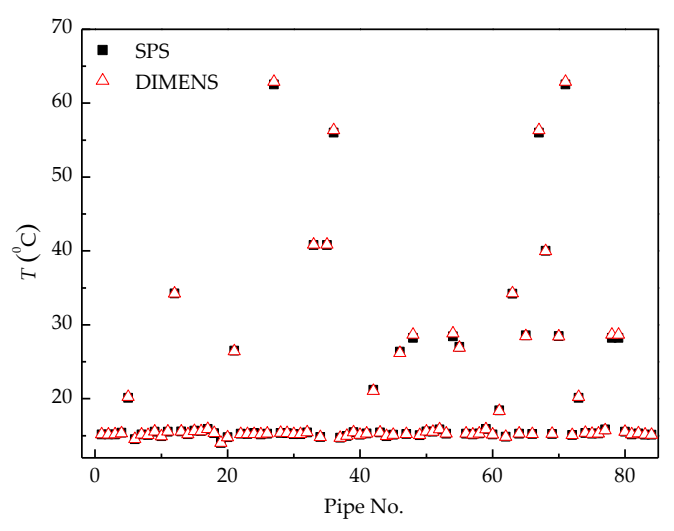

(c)

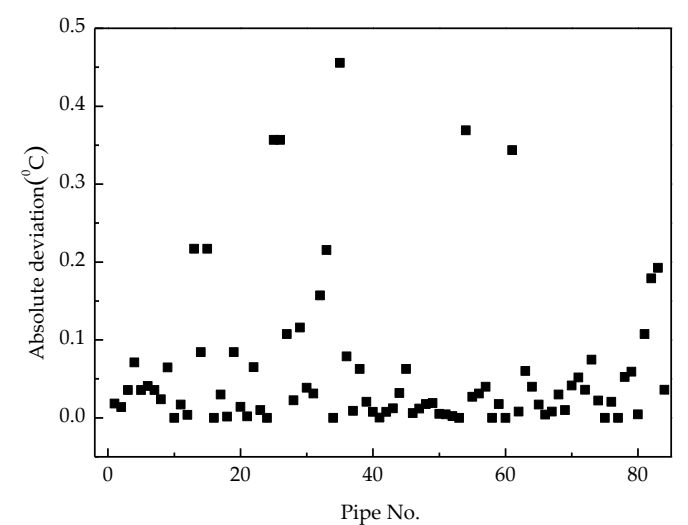

(b)

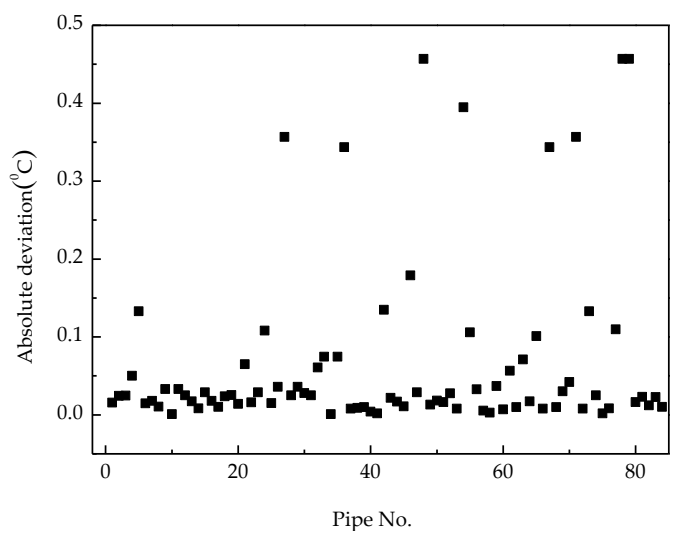

(d)

Figure 13. Comparison of temperature at the start and end nodes of pipelines: (a) Temperature value at the start node; (b) deviation at the start node; (c) temperature value at end node; (d) deviation at the end node.

\subsection{Comparison of Computational Efficiency}

The number of discretized grid points of the pipeline network and the number of pipeline sections of the pipeline network are two main factors that can affect the efficiency of pipeline network simulation. Therefore, the computational speed of the DIMENS method proposed in this paper is examined from three aspects: (1) the number of discretized grid points; (2) the number of components; and (3) the number of discretized grid points and the number of components. The restart process of the complex pipeline network is simulated by both the DIMENS method and SPS, and the CPU times are recorded and compared. The time step is $10 \mathrm{~s}$, and the simulation time is 1 day. The computations are carried out on a computer equipped with a $2.6 \mathrm{GHz}$ Intel Xeon E5-2640 CPU with 64 GB RAM.

First, the topology of the pipeline network in Figure 5 is unchanged, while the pipelines are discretized by different spatial steps. The adaptability of the DIMENS method for the number of discretized grid points is studied. The CPU time is shown in Figure 14.

It can be seen from Figure 14 that the CPU time of the DIMENS method is less than that of the SPS. This means that the DIMENS method is more efficient than SPS. What is more, the CPU times of these two methods are both almost linear with the number of discretized points. This indicates that the DIMNES method and the SPS both have a strong adaptability to the increase of discretized points of the pipeline network. However, with the same number of discretized points, the CPU time of SPS is always longer than that of DIMENS method. The slope of the line of the DIMENS method in Figure 14 
is 0.01 , while that of SPS is 0.036 . This is means that the efficiency of the DIMENS method is about $0.0036 / 0.01=3.6$ times higher than that of the SPS as the number of discretized points is increasing. In other word, the DIMENS method is more efficient than SPS.

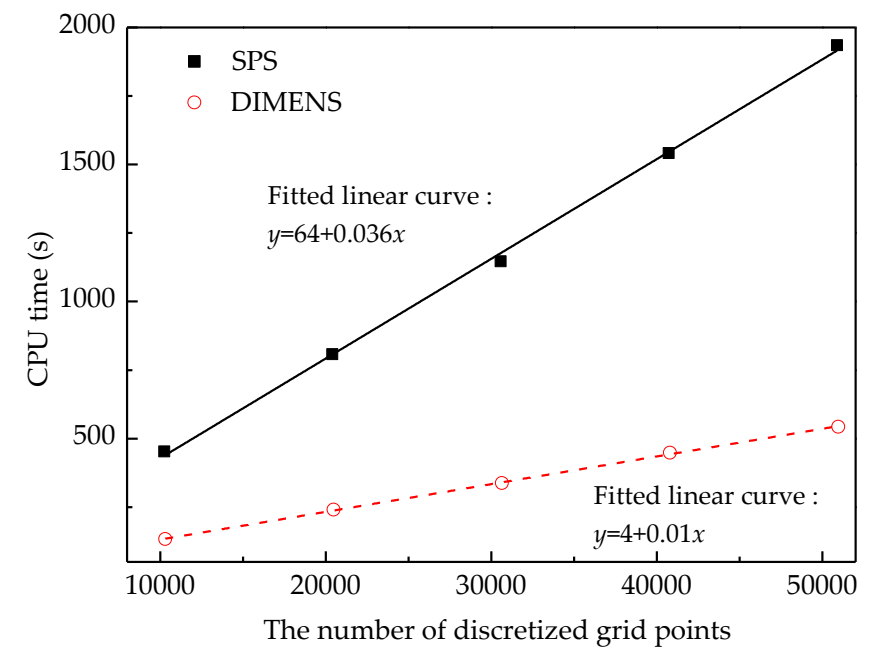

Figure 14. Change of CPU time with the number of discretized grid points.

Second, the total number of discretized grid points is unchanged, while the number of components of the pipeline network is increased. The adaptability of the DIMENS method for the number of components is studied. The CPU time is shown in Figure 15.

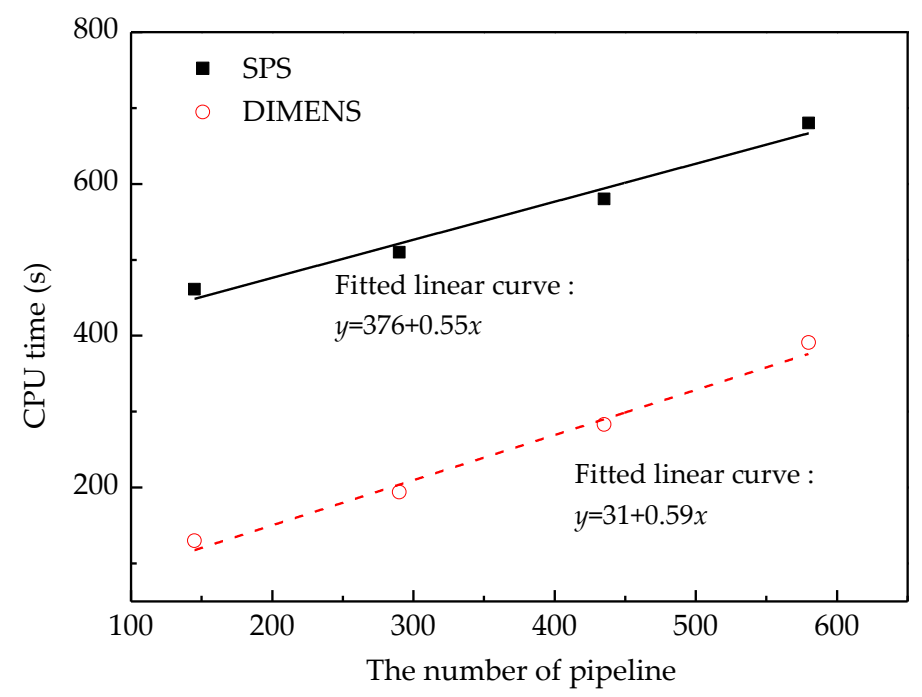

Figure 15. Change of computing time with the number of pipeline sections.

It is clearly shown in Figure 15 that the CPU time of the DIMENS method is also less than that of the SPS. This means that the computational efficiency of the DIMENS method is high. What is more, the CPU time of the DIMENS method and that of the SPS are both linear with the number of components. The fit linear curve of SPS is $y=376+0.55 x$, and that of DIMENS is $y=31+0.59 x$. In other words, the gradients of the two lines are similar. Thus, the DIMNES method and the SPS are both not only well adapted to the increase of discrete points, but also to the increase of components. This indicates that the DIMNES method and the SPS both have a strong adaptability to the number of pipeline sections. 
Last, the pipe network, as shown in Figure 5, is copied multiple times and connected to each other to form a larger network. The adaptability of the DIMENS method for the couple of grid points and components is studied. The CPU time is shown in Figure 16.

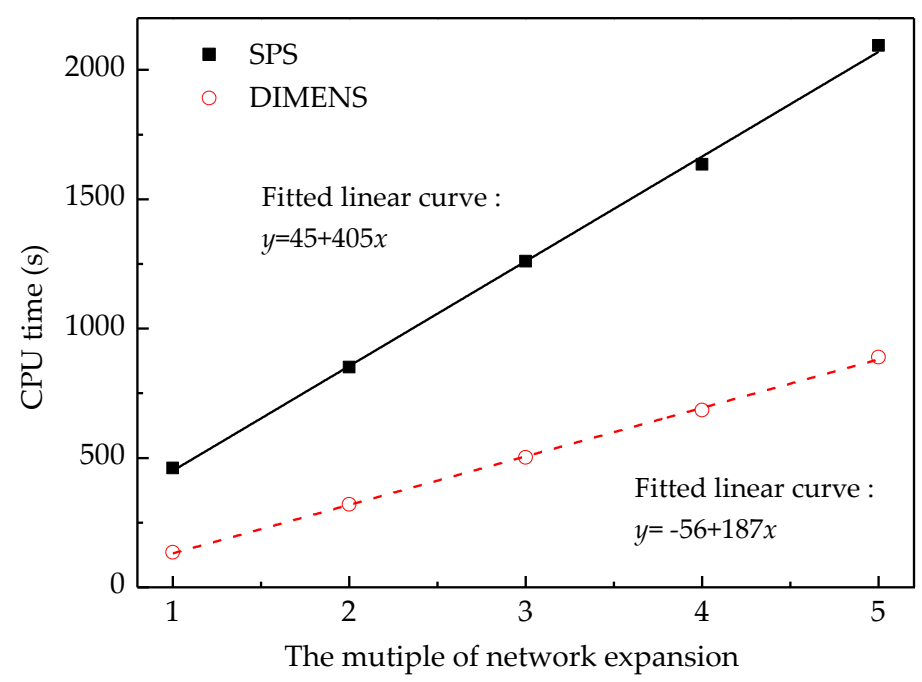

Figure 16. Change of computing time with multiple network expansions.

Figure 16 shows that the CPU time of the DIMENS method and that of the SPS are both linear with multiple network expansions. However, with the same multiple network expansions, the CPU time of SPS is always more than that of DIMENS method, and the slope of the line of the DIMENS method is smaller than that of SPS. That is to say, the CPU time of the DIMENS method is always less than that of SPS, and the DIMENS method would be more time-saving with the larger scale of the pipe network. This indicates that the DIMENS method is more efficient than SPS. It can be further seen that the fitted linear curve of SPS is $y=45+405 x$, while that of DIMENS is $y=-56+187 x$. In other words, the slope of the line of the DIMENS method is about 187, while the slope of the line of SPS is about 405, which is over two times greater $(405 / 187=2.16)$ than that of the DIMENS method. This means that the computing efficiency of the DIMENS method is over two times greater than that of SPS.

\section{Conclusions and Future Work}

In this paper, the DIMENS method has been extended to the flow and thermodynamic-coupled simulation of the complex pipeline network that includes pipelines and non-pipeline components. The simulation accuracy and efficiency of the present method have been investigated through an example of the complex pipeline network. The conclusions are as follows:

(1) DIMES shows a very good agreement with SPS (differences below 3.5\%). This accuracy can meet the requirements of practical engineering;

(2) The CPU time of the DIMENS method is always less than that of SPS, and the computational speed of the DIMENS method is over two times higher than that of the SPS;

(3) The DIMENS method has a strong adaptability to the scale of the pipeline network. The CPU time of the DIMNES method is linear with the size of the pipeline network.

In recent years, as a rapidly developing role in High Performance Computing (HPC), Graphic Processing Unit (GPU) computing has been becoming a research hotspot in many research fields. In order to maximize the GPU computing speed, the parallel granularity of the computational task should be fine enough. Thus, the algorithm should be highly parallel. In the DIMENS method, the pipelines in the network are parallel and the solution process of one pipeline is parallel too. This means that the parallel characteristic of the DIMENS method greatly matches the GPU. Therefore, 
to improve the computational speed further, it would be meaningful and worthwhile to study the GPU-accelerated simulation for large-scale natural gas pipeline networks in the future.

Author Contributions: Conceptualization, B.Y.; Methodology, P.W.; Validation, S.A., D.H., and Y.X.; Writing—original draft, P.W.; Writing—review \& editing, P.W. and B.Y.

Funding: The study is supported by the National Natural Science Foundation of China (No. 518060180), the Project of Construction of Innovative Teams and Teacher Career Development for Universities and Colleges under Beijing Municipality (No. IDHT20170507), and the Program of Great Wall Scholar (No. CIT\&TCD20180313).

Conflicts of Interest: The authors declare no conflict of interest.

\section{Nomenclature}

\section{Roman symbols}

$\begin{array}{ll}c_{\mathrm{V}} & \text { Specific heat capacity at constant volume, } \mathrm{J} / \mathrm{kg} \cdot \mathrm{K}) \\ c_{\mathrm{p}} & \text { Specific heat capacity at constant pressure, } \mathrm{J} / \mathrm{kg} \cdot \mathrm{K}) \\ e & \text { Specific internal energy, } \mathrm{J} /(\mathrm{kg} \cdot \mathrm{K}) \\ d & \text { Internal diameter of pipe, } \mathrm{m} \\ g & \text { Gravitational acceleration, } \mathrm{m} / \mathrm{s}^{2} \\ h & \text { Specific enthalpy, } \mathrm{J} / \mathrm{kg} \cdot \mathrm{K}) \\ m & \text { Mass flow rate, } \mathrm{kg} / \mathrm{s} \\ n & \text { Polytropic index } \\ p & \text { Pressure, Pa } \\ s & \text { Elevation, } \mathrm{m} \\ t & \text { Time, } \mathrm{s} \\ u & \text { Corresponding component of general variable } \mathrm{U} \\ w & \text { Flow velocity, } \mathrm{m} / \mathrm{s} \\ x & \text { Spatial coordinate, } \mathrm{m} \\ A & \text { Cross-section area, } \mathrm{m}^{2} \\ C_{\mathrm{V}} & \text { Flow coefficient of valve } \\ F R & \text { Valve opening } \\ \mathrm{K} & \text { Total heat transfer coefficient, } \mathrm{W} /\left(\mathrm{m}^{2} \cdot \mathrm{K}\right) \\ N & \text { Number of discretized sections of pipeline } \\ Q & \text { Volume flow, } \mathrm{m}^{3} / \mathrm{s} \\ R & \text { Specific gas constant, } \mathrm{J} /(\mathrm{kg} \cdot \mathrm{K}) \\ T & \text { Temperature, } \mathrm{K} \\ \mathrm{U} & \text { General variable } \\ \mathrm{Z} & \text { Compressibility factor } \\ & \end{array}$

\section{Greek symbols}

$\begin{array}{ll}\alpha, \beta & \text { Fundamental set of solution } \\ \gamma & \text { Particular solution } \\ \rho & \text { Density, } \mathrm{kg} / \mathrm{m}^{3} \\ \theta & \text { Inclination angle of a pipe, rad } \\ \lambda & \text { Friction factor } \\ \varepsilon & \text { Compression ratio } \\ \Delta & \text { Specific density of natural gas relative to air } \\ \Delta x & \text { Spatial step, } \mathrm{m} \\ \Delta t & \text { Time step, } \mathrm{s}\end{array}$

\section{Superscripts}

$\begin{array}{ll}n & \text { Time level } \\ m & \text { Related to mass flow } \\ p & \text { Related to pressure }\end{array}$




\section{Subscripts}

$\begin{array}{ll}\text { npc } & \text { Non-pipeline components } \\ \text { in } & \text { Flow in } \\ \text { out } & \text { Flow out } \\ \text { p } & \text { Pipeline } \\ \text { c } & \text { Compressor } \\ \text { v } & \text { Valve } \\ \text { s } & \text { Supply } \\ \text { d } & \text { Demand }\end{array}$

\section{Appendix A}

The pipeline model is a set of governing equations of homogeneous, geometrically one-dimensional flow in the pipeline, which consists of the continuity equation (A1), momentum equation (A2), and energy equation (A3):

$$
\begin{gathered}
\frac{\partial \rho}{\partial t}+\frac{\partial(\rho w)}{\partial x}=0 \\
\frac{\partial(\rho w)}{\partial t}+\frac{\partial(\rho w w)}{\partial x}+\frac{\partial p}{\partial x}=-\frac{\lambda}{2} \frac{\rho w|w|}{d}-\rho g \sin \theta, \\
\frac{\partial}{\partial t}\left[\left(e+\frac{w^{2}}{2}+g s\right) \rho A\right]+\frac{\partial}{\partial x}\left[\left(h+\frac{w^{2}}{2}+g s\right) \rho w A\right]=-q \rho w A,
\end{gathered}
$$

With the following equations:

Cross-section area:

$$
A=\frac{\pi}{4} d^{2}
$$

Mass flux:

$$
m=\rho w A,
$$

Specific heat increment:

$$
q=\frac{\pi K d\left(T-T_{g}\right)}{m}
$$

Relationship between elevation and inclination:

$$
\theta=\frac{\partial s}{\partial x}
$$

Derivation rule of composite functions:

$$
\begin{gathered}
\frac{\partial \rho}{\partial t}=\left(\frac{\partial \rho}{\partial p}\right)_{T} \frac{\partial p}{\partial t}+\left(\frac{\partial \rho}{\partial T}\right)_{p} \frac{\partial T}{\partial t}, \\
\frac{\partial \rho}{\partial x}=\left(\frac{\partial \rho}{\partial p}\right)_{T} \frac{\partial p}{\partial x}+\left(\frac{\partial \rho}{\partial T}\right)_{p} \frac{\partial T}{\partial x}, \\
\frac{d(\cdot)}{d t}=\frac{\partial(\cdot)}{\partial t}+w \frac{\partial(\cdot)}{\partial x},
\end{gathered}
$$

Fundamental equations of thermodynamic functions:

$$
\begin{gathered}
\left(\frac{\partial \rho}{\partial T}\right)_{p}=-\left(\frac{\partial p}{\partial T}\right)_{\rho}\left(\frac{\partial \rho}{\partial p}\right)_{T}=-\frac{\left(\frac{\partial p}{\partial T}\right)_{\rho}}{\left(\frac{\partial p}{\partial \rho}\right)_{T}} \\
\left(\frac{\partial \rho}{\partial p}\right)_{T}=\frac{1}{\left(\frac{\partial p}{\partial \rho}\right)_{T}}
\end{gathered}
$$




$$
\begin{gathered}
d h=d e+d\left(\frac{p}{\rho}\right), \\
d e=c_{v} d T-\frac{1}{\rho^{2}}\left[T\left(\frac{\partial p}{\partial T}\right)_{\rho}-p\right] d \rho,
\end{gathered}
$$

Equations (A1) to (A3) can be transformed to mathematical forms with pressure, mass rate, and temperature as the dependent variables.

\section{Continuity Equation}

Substituting Equation (A5) and Equation (A8) into Equation (A1),

$$
\left(\frac{\partial \rho}{\partial p}\right)_{T} \frac{\partial p}{\partial t}+\left(\frac{\partial \rho}{\partial T}\right)_{p} \frac{\partial T}{\partial t}+\frac{1}{A} \frac{\partial m}{\partial x}=0
$$

Substituting Equation (A11) and Equation (A12) into Equation (A13),

$$
\frac{\partial p}{\partial t}-\left(\frac{\partial p}{\partial T}\right)_{\rho} \frac{\partial T}{\partial t}+\frac{1}{A}\left(\frac{\partial p}{\partial \rho}\right)_{T} \frac{\partial m}{\partial x}=0
$$

\section{Momentum Equation}

Substituting Equation (A5) into Equation (A2),

$$
\frac{\partial m}{\partial t}+\frac{2 m}{A \rho} \frac{\partial m}{\partial x}-\frac{m^{2}}{A \rho^{2}} \frac{\partial \rho}{\partial x}+A \frac{\partial p}{\partial x}=-\frac{\lambda}{2} \frac{m|m|}{d A \rho}-A \rho g \sin \theta
$$

Substituting Equation (A9) into Equation (A17),

$$
\frac{\partial m}{\partial t}+\left[A-\frac{m^{2}}{A \rho^{2}}\left(\frac{\partial \rho}{\partial p}\right)_{T}\right] \frac{\partial p}{\partial x}+\frac{2 m}{A \rho} \frac{\partial m}{\partial x}=-\frac{\lambda}{2} \frac{m|m|}{d A \rho}-A \rho g \sin \theta+\frac{m^{2}}{A \rho^{2}}\left(\frac{\partial \rho}{\partial T}\right)_{p} \frac{\partial T}{\partial x}
$$

\section{Energy Equation}

Substituting Equations (A4)-(A7) into Equation (A3),

$$
\frac{\partial}{\partial t}\left[\left(h+\frac{w^{2}}{2}\right) \rho A\right]-\frac{\partial p}{\partial t}+\frac{\partial}{\partial x}\left[\left(h+\frac{w^{2}}{2}\right) \rho w A\right]=-\pi K d\left(T-T_{g}\right)-\rho w g A \sin \theta,
$$

Substituting Equation (A13) into Equation (A19),

$$
\frac{\partial}{\partial t}\left[\left(h+\frac{w^{2}}{2}\right) \rho\right]-\frac{\partial p}{\partial t}+w \frac{\partial}{\partial x}\left[\left(h+\frac{w^{2}}{2}\right) \rho\right]+\left(h+\frac{w^{2}}{2}\right) \rho \frac{\partial w}{\partial x}=-\frac{4 K\left(T-T_{g}\right)}{d}-\rho w g \sin \theta
$$

Equation (A20) can be rewritten in the form

$$
\begin{gathered}
\rho \frac{d}{d t}\left(h+\frac{w^{2}}{2}\right)+\left(h+\frac{w^{2}}{2}\right)\left[\frac{\partial \rho}{\partial t}+w \frac{\partial \rho}{\partial x}+\rho \frac{\partial w}{\partial x}\right]-\frac{\partial p}{\partial t}=-\frac{4 K\left(T-T_{g}\right)}{d}-\rho w g \sin \theta, \\
\rho \frac{d h}{d t}-\frac{\partial p}{\partial t}+\rho w \frac{d w}{d t}=-\frac{4 K\left(T-T_{g}\right)}{d}-\rho w g \sin \theta,
\end{gathered}
$$

Equation (A2) can be rewritten in the form

$$
\begin{gathered}
\rho \frac{\partial w}{\partial t}+\rho w \frac{\partial w}{\partial x}+\frac{\partial p}{\partial x}=-\frac{\lambda}{2} \frac{\rho w|w|}{d}-\rho g \sin \theta, \\
\rho w \frac{d w}{\partial t}=-w \frac{\partial p}{\partial x}-\frac{\lambda}{2} \frac{\rho|w|^{3}}{d}-\rho w g \sin \theta,
\end{gathered}
$$

Substituting Equation (A22b) into Equation (A21b),

$$
\rho \frac{d h}{d t}-\frac{\partial p}{\partial t}-w \frac{\partial p}{\partial x}=\frac{\lambda}{2} \frac{\rho|w|^{3}}{d}-\frac{4 K\left(T-T_{g}\right)}{d}
$$




$$
\rho \frac{d h}{d t}-\frac{d p}{d t}=\frac{\lambda}{2} \frac{\rho|w|^{3}}{d}-\frac{4 K\left(T-T_{g}\right)}{d},
$$

Substituting Equation (A13) into Equation (A23b),

$$
\rho \frac{d e}{d t}-\frac{p}{\rho} \frac{d \rho}{d t}=\frac{\lambda}{2} \frac{\rho|w|^{3}}{d}-\frac{4 K\left(T-T_{g}\right)}{d},
$$

Substituting Equation (A14) into Equation (A24),

$$
\rho c_{v} \frac{d T}{d t}-T\left(\frac{\partial p}{\partial T}\right)_{\rho} \frac{1}{\rho} \frac{d \rho}{d t}=\frac{\lambda}{2} \frac{\rho|w|^{3}}{d}-\frac{4 K\left(T-T_{g}\right)}{d},
$$

Equation (A1) can be rewritten in the form

$$
\begin{gathered}
\frac{\partial \rho}{\partial t}+w \frac{\partial \rho}{\partial x}+\rho \frac{\partial w}{\partial x}=0, \\
\frac{d \rho}{d t}=-\rho \frac{\partial w}{\partial x},
\end{gathered}
$$

Substituting Equation (A26b) into Equation (A25),

$$
\frac{\partial T}{\partial t}+w \frac{\partial T}{\partial x}=\frac{1}{\rho c_{v}}\left[-T\left(\frac{\partial p}{\partial T}\right) \frac{\partial w}{\rho x}+\frac{\lambda}{2} \frac{\rho|w|^{3}}{d}-\frac{4 K\left(T-T_{g}\right)}{d}\right],
$$

Equation (A14), Equation (16) and Equation (A23) can be written in the general form

$$
\frac{\partial \mathbf{U}}{\partial t}+\mathbf{B} \cdot \frac{\partial \mathbf{U}}{\partial x}=\mathbf{F}
$$

The detailed expressions of $\mathbf{U}, \mathbf{G}$, and $\mathbf{S}$ in the pipeline model are as follows:

Flow Equation:

$$
\begin{aligned}
& \mathbf{U}=\left[\begin{array}{l}
u_{1} \\
u_{2}
\end{array}\right]=\left[\begin{array}{c}
p \\
m
\end{array}\right], \\
& \mathbf{G}=\left[\begin{array}{cc}
\frac{1}{A} \frac{\partial}{\partial p}\left(\frac{\partial p}{\partial \rho}\right)_{T} \frac{\partial m}{\partial x} & 0 \\
\left\{\begin{array}{c}
\frac{m^{2}}{A}\left[\frac{2}{\rho^{3}}\left(\frac{\partial \rho}{\partial p}\right)_{T}^{2}-\frac{1}{\rho^{2}} \frac{\partial}{\partial p}\left(\frac{\partial \rho}{\partial p}\right)_{T}\right] \frac{\partial p}{\partial x} \\
-\frac{2 m}{A \rho^{2}}\left(\frac{\partial \rho}{\partial p}\right)_{T} \frac{\partial m}{\partial x}
\end{array}\right\} & -\frac{2 m}{A \rho^{2}}\left(\frac{\partial \rho}{\partial p}\right)_{T} \frac{\partial p}{\partial x}+\frac{2}{A \rho} \frac{\partial m}{\partial x}
\end{array}\right], \\
& \mathbf{S}=\left[\begin{array}{cc}
\frac{\partial T}{\partial t} \frac{\partial}{\partial p}\left(\frac{\partial p}{\partial T}\right)_{\rho} & 0 \\
\left\{\begin{array}{c}
\lambda \frac{m|m|}{2}\left(\frac{\partial \rho}{\partial p}\right)_{T}-A g \sin \theta\left(\frac{\partial \rho}{\partial p}\right)_{T} \\
+\frac{m^{2}}{A} \frac{\partial T}{\partial x} \frac{\partial}{\partial p}\left(\frac{1}{\rho^{2}}\left(\frac{\partial \rho}{\partial T}\right)_{p}\right)
\end{array}\right. & -\lambda \frac{|m|}{d A \rho}+\frac{2 m}{A \rho^{2}}\left(\frac{\partial \rho}{\partial T}\right)_{p} \frac{\partial T}{\partial x}
\end{array}\right],
\end{aligned}
$$

Thermodynamic Equation:

$$
\begin{gathered}
U=T \\
G=0 \\
S=\left\{\begin{array}{c}
\frac{1}{\rho c_{v}}\left(-\left(\frac{\partial p}{\partial T}\right) \frac{\partial w}{\partial x}-T \frac{\partial w}{\partial x} \frac{\partial}{\partial T}\left(\frac{\partial p}{\partial T}\right)_{\rho}+\frac{\lambda}{2} \frac{|w|^{3}}{d} \frac{\partial \rho}{\partial T}-\frac{4 K}{d}\right) \\
-\left[-T\left(\frac{\partial p}{\partial T}\right) \frac{\partial w}{\partial x}+\frac{\lambda}{2} \frac{\rho|w|^{3}}{d}-\frac{4 K\left(T-T_{\mathrm{a}}\right)}{d}\right]\left(\frac{1}{\rho c_{v}}\right)^{2}\left(\rho \frac{\partial c_{v}}{\partial T}+c_{v} \frac{\partial \rho}{\partial T}\right)
\end{array}\right\},
\end{gathered}
$$

\section{Appendix B}

To describe in detail how the flow equations of multi-component interconnection nodes are solved, a pipeline network is given as the instance. The pipeline network is comprised of three pipes, one compressor, one valve, and two externals, as shown in Figure A1. The closed equations for the solution of multi-component interconnection nodes are Equations (A35)-(A58), shown as below. 


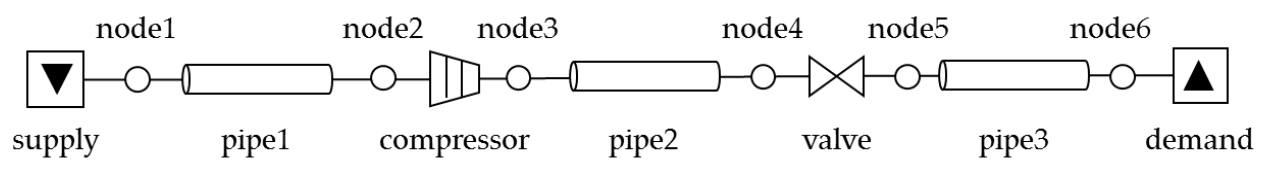

Figure A1. The schematic diagram of the structure of a pipeline network.

Flow equations of the compressor:

$$
\begin{aligned}
& m_{\mathrm{in}, \mathrm{c}}-m_{\mathrm{out}, \mathrm{c}}=0, \\
& \varepsilon p_{\mathrm{in}, \mathrm{c}}-p_{\mathrm{out}, \mathrm{c}}=0
\end{aligned}
$$

Flow equations of the valve:

$$
\begin{gathered}
m_{\mathrm{in}, \mathrm{v}}-m_{\mathrm{out}, \mathrm{v}}=0, \\
m_{\mathrm{in}, \mathrm{v}}-C_{v} \rho_{\mathrm{in}, \mathrm{v}} \sqrt{\frac{\left(p_{\mathrm{in}, \mathrm{v}}^{2}-p_{\mathrm{out}, \mathrm{v}}^{2}\right)}{Z \Delta \overline{T_{\mathrm{in}, \mathrm{v}}}}}=0,
\end{gathered}
$$

Flow equation of supply:

$$
p_{\mathrm{s}}=p(t),
$$

Flow equation of demand:

$$
m_{\mathrm{d}}=m(t)
$$

Flow equations of multi-component interconnection node 1:

$$
\begin{aligned}
& m_{\mathrm{s}}=m_{1, \mathrm{p} 1}, \\
& p_{\mathrm{s}}=p_{1, \mathrm{p} 1},
\end{aligned}
$$

Flow equations of multi-component interconnection node 2 :

$$
\begin{aligned}
& m_{N_{1}+1, \mathrm{p} 1}=m_{\mathrm{in}, \mathrm{c}} \\
& p_{N_{1}+1, \mathrm{p} 1}=p_{\mathrm{in}, \mathrm{c}}
\end{aligned}
$$

Flow equations of multi-component interconnection node 3 :

$$
\begin{aligned}
& m_{\text {out }, \mathrm{c}}=m_{1, \mathrm{p} 2} \\
& p_{\text {out }, \mathrm{c}}=p_{1, \mathrm{p} 2},
\end{aligned}
$$

Flow equations of multi-component interconnection node 4 :

$$
\begin{aligned}
& m_{N_{2}+1, \mathrm{p} 2}=m_{\mathrm{in}, \mathrm{v}}, \\
& p_{N_{2}+1, \mathrm{p} 2}=p_{\mathrm{in}, \mathrm{v}},
\end{aligned}
$$

Flow equations of multi-component interconnection node 5:

$$
\begin{gathered}
m_{\mathrm{out}, \mathrm{v}}=m_{1, \mathrm{p} 3}, \\
p_{\mathrm{in}, \mathrm{v}}=p_{1, \mathrm{p} 3},
\end{gathered}
$$

Flow equations of multi-component interconnection node 6 :

$$
\begin{aligned}
& m_{N_{3}+1, \mathrm{p} 3}=m_{\mathrm{d}}, \\
& p_{N_{3}+1, \mathrm{p} 3}=p_{\mathrm{d}},
\end{aligned}
$$


Supplemental equations of pipe 1:

$$
\begin{gathered}
m_{1, \mathrm{p} 1}=\alpha_{1, \mathrm{p} 1}^{m} p_{1, \mathrm{p} 1}+\beta_{1, \mathrm{p} 1}^{m} m_{N_{1}+1, \mathrm{p} 1}+\gamma_{1, \mathrm{p} 1}^{m} \\
p_{N_{1}+1, \mathrm{p} 1}=\alpha_{\mathrm{N}_{1}+1, \mathrm{p} 1}^{p} p_{1, \mathrm{p} 1}+\beta_{\mathrm{N}_{1}+1, \mathrm{p} 1}^{p} m_{N_{1}+1, \mathrm{p} 1}+\gamma_{N_{1}+1, \mathrm{p} 1}^{p},
\end{gathered}
$$

Supplemental equations of pipe 2:

$$
\begin{gathered}
m_{1, \mathrm{p} 2}=\alpha_{1, \mathrm{p} 2}^{m} p_{1, \mathrm{p} 2}+\beta_{1, \mathrm{p} 2}^{m} m_{N_{2}+1, \mathrm{p} 2}+\gamma_{1, \mathrm{p} 2}^{m} \\
p_{N_{2}+1, \mathrm{p} 2}=\alpha_{\mathrm{N}_{2}+1, \mathrm{p} 2}^{p} p_{1, \mathrm{p} 2}+\beta_{N_{2}+1, \mathrm{p} 2}^{p} m_{N_{2}+1, \mathrm{p} 2}+\gamma_{N_{2}+1, \mathrm{p} 2}^{p}
\end{gathered}
$$

Supplemental equations of pipe 3:

$$
\begin{gathered}
m_{1, \mathrm{p} 3}=\alpha_{1, \mathrm{p} 3}^{m} p_{1, \mathrm{p} 3}+\beta_{1, \mathrm{p} 3}^{m} m_{N_{3}+1, \mathrm{p} 3}+\gamma_{1, \mathrm{p} 3}^{m}{ }^{\prime \prime} \\
p_{N_{3}+1, \mathrm{p} 3}=\alpha_{N_{3}+1, \mathrm{p} 3}^{p} p_{1, \mathrm{p} 3}+\beta_{N_{3}+1, \mathrm{p} 3}^{p} m_{N_{3}+1, \mathrm{p} 3}+\gamma_{N_{3}+1, \mathrm{p} 3}^{p}
\end{gathered}
$$

The number of the mass flow and pressure variables at the starting and terminal points of the pipeline is 4 , and the numbers of the compressor and valve are both 4 too, while the number of the mass flow and pressure variables of the external component is 2 . This means that there are a total of $4 \times 3+4 \times 1+4 \times 1+2 \times 2=24$ unknown variables for solving the multi-component interconnection nodes in the pipeline network in Figure 5 . The number of equations in Equations (A35)-(A58) is 24. Therefore, these equations are closed and have a unique solution.

\section{Appendix C}

To describe how the thermodynamic equations of multi-component interconnection nodes are solved in detail, a pipeline network is given as the instance. For instance, the flow state of the pipeline network at a certain moment is shown in Figure A2. Pipe 1, pipe 2, and pipe 3 are respectively the second, fourth, and first type of pipeline. The complete system of linear equations of the multi-component interconnection nodes is presented as follows:

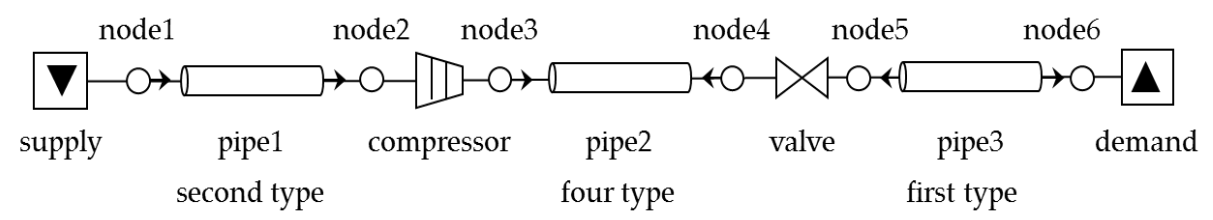

Figure A2. The flow status of the pipeline networks at a given time.

Thermodynamic-linearized equation of the compressor:

$$
T_{\text {out }, \mathrm{c}}-T_{\mathrm{in}, \varepsilon^{2}} \varepsilon^{\frac{m-1}{m}}=0,
$$

Thermodynamic equation of the valve:

$$
T_{\mathrm{out}, \mathrm{v} 1}-T_{\mathrm{in}, \mathrm{v} 1}+\left(p_{\mathrm{out}, \mathrm{v} 1}-p_{\mathrm{in}, \mathrm{v} 1}\right) \frac{1}{c_{p}}\left[\frac{T}{\rho^{2}} \frac{(\partial p / \partial T)_{\rho}}{(\partial p / \partial \rho)_{T}}-\frac{1}{\rho}\right]_{\mathrm{out}, \mathrm{v} 1}=0
$$

Thermodynamic equation of the external component:

$$
T_{\mathrm{S}}=T_{1}(t),
$$

Thermodynamic equations of multi-component interconnection nodes 1:

$$
T_{1, \mathrm{p} 1}=\frac{\left|c_{p} m\right|_{\mathrm{e} 1}}{\left|c_{p} m\right|_{1, \mathrm{p} 1}} T_{\mathrm{s}},
$$


Thermodynamic equations of multi-component interconnection nodes 2:

$$
T_{\mathrm{in}, \mathrm{c}}=\frac{\left|c_{p} m\right|_{N_{1}+1, \mathrm{p} 1}}{\left|c_{p} m\right|_{\mathrm{in}, \mathrm{c}}} T_{N_{1}+1, \mathrm{p} 1}
$$

Thermodynamic equations of multi-component interconnection nodes 3:

$$
T_{1, \mathrm{p} 2}=\frac{\left|c_{p} m\right|_{\text {out, } \mathrm{c}}}{\left|c_{p} m\right|_{1, \mathrm{p} 2}} T_{\text {out }, \mathrm{c}}
$$

Thermodynamic equations of multi-component interconnection nodes 4 :

$$
T_{N_{2}+1, \mathrm{p} 2}=\frac{\left|c_{p} m\right|_{\mathrm{in}, v}}{\left|c_{p} m\right|_{N_{2}+1, \mathrm{p} 2}} T_{\mathrm{in}, v}
$$

Thermodynamic equations of multi-component interconnection nodes 5 :

$$
T_{1, \mathrm{p} 3}=\frac{\left|c_{p} m\right|_{\text {out }, \mathrm{v}}}{\left|c_{p} m\right|_{1, \mathrm{p} 3}} T_{\mathrm{out}, \mathrm{v}}
$$

Thermodynamic equations of multi-component interconnection nodes 6:

$$
T_{\mathrm{d}}=\frac{\left|c_{p} m\right|_{N_{3}+1, \mathrm{p} 3}}{\left|c_{p} m\right|_{2 \mathrm{~g}}} T_{N_{3}+1, \mathrm{p} 3}
$$

Temperature value of the starting and terminal points of pipe 3:

$$
\begin{gathered}
T_{1, \mathrm{p} 3}=T_{1, \mathrm{p} 3}^{\text {solved }}, \\
T_{N+1, \mathrm{p} 3}=T_{N+1, \mathrm{p} 3}^{\text {solved }}
\end{gathered}
$$

where, the superscript 'solved' is standing for having been solved in the second step.

Supplemental equation of pipe 1:

$$
T_{N_{1}+1, \mathrm{p} 1}=T_{1, \mathrm{p} 1} \alpha_{N_{1}+1, \mathrm{p} 1}+\gamma_{N_{1}+1, \mathrm{p} 1}
$$

The number of temperature variables at the starting and terminal points of the pipeline, compressor, and valve is 2 , while the number of temperature variables of the external component is 1 . This means that there are $5 \times 2+2 \times 1=12$ unknown temperature variables for solving the multi-component interconnection nodes solution in pipeline networks, as shown in Figure A2. There are also 12 Equations in Equations (A59) to (A70). Therefore, these equations are closed and have a unique solution.

\section{References}

1. Birol, F. Golden Rules for a Golden Age of Gas: World Energy Outlook Special Report on Unconventional Gas: Report. International Energy Agency. Available online: www.worldenergyoutlook.org/media/weowebsite/ 2012/goldenrules/WEO2012_GoldeGRulesReport.pdf (accessed on 22 October 2012).

2. Turner, W.J.; Kwon, P.S.J.; Maguire, P.A. Evaluation of a gas pipeline simulation program. Math. Comput. Model. 1991, 15, 1-14. [CrossRef]

3. Ouellette, L.; Hanks, H. Integrated simulation and SCADA to replay historical data for a local distribution company. In Proceedings of the PSIG Annual Meeting, Pipeline Simulation Interest Group, Tucson, AZ, USA, 15-17 October 1997.

4. Hendriks, P.H.G.M.; Postvoll, W.; Mathiesen, M.; Spiers, R.P.; Siddorn, J. Improved capacity utilization by integrating real-time sea bottom temperature data. In Proceedings of the PSIG annual Meeting, Pipeline Simulation Interest Group, Williamsburg, VA, USA, 23-25 October 1995. 
5. Osiadacz, A. Simulation and Analysis of Gas Networks; E. \& F.N. Spon Ltd.: London, UK, 1987; pp. 69-82.

6. Helgaker, J.F.; Müller, B.; Ytrehus, T. Transient flow in natural gas pipelines using implicit finite difference schemes. J. Offshore Mech. Arct. Eng. 2014, 136, 031701. [CrossRef]

7. Wang, H.; Liu, X.; Zhou, W. Transient flow simulation of municipal gas pipelines and networks using semi implicit finite volume method. Procedia Eng. 2011, 12, 217-223.

8. Ebrahimzadeh, E.; Shahrak, M.N.; Bazooyar, B. Simulation of transient gas flow using the orthogonal collocation method. Chem. Eng. Res. Des. 2012, 90, 1701-1710. [CrossRef]

9. Alamian, R.; Behbahani-Nejad, M.; Ghanbarzadeh, A. A state space model for transient flow simulation in natural gas pipelines. J. Nat. Gas Sci. Eng. 2012, 9, 51-59. [CrossRef]

10. Behbahani-Nejad, M.; Shekari, Y. The accuracy and efficiency of a reduced-order model for transient flow analysis in gas pipelines. J. Pet. Sci. Eng. 2010, 73, 13-19. [CrossRef]

11. Farzaneh-Gord, M.; Rahbari, H.R. Unsteady natural gas flow within pipeline network, an analytical approach. J. Nat. Gas Sci. Eng. 2016, 28, 397-409. [CrossRef]

12. Pambour, K.A.; Bolado-Lavin, R.; Dijkema, G.P.J. An integrated transient model for simulating the operation of natural gas transport systems. J. Nat. Gas Sci. Eng. 2016, 28, 672-690. [CrossRef]

13. Madoliat, R.; Khanmirza, E.; Moetamedzadeh, H.R. Transient simulation of gas pipeline networks using intelligent methods. J. Nat. Gas Sci. Eng. 2016, 29, 517-529. [CrossRef]

14. Wang, J.; Wang, T.; Wang, J. Application of $\pi$ equivalent circuit in mathematic modeling and simulation of gas pipeline. Appl. Mech. Mater. 2014, 496, 943-946. [CrossRef]

15. Herrán-González, A.; Cruz, J.M.D.L.; De Andrés-Toro, B.; Risco-Martín, J.L. Modeling and Simulation of a Gas Distribution Pipeline Network. Appl. Math. Model. 2009, 33, 1584-1600. [CrossRef]

16. Thorley, A.R.D.; Tiley, C.H. Unsteady and transient flow of compressible fluids in pipelines-A review of theoretical and some experimental studies. Int. J. Heat Fluid Flow 1987, 8, 3-15. [CrossRef]

17. Kiuchi, T.T.; Izumi, H.H.; Huke, T.T. An operation support system of large city gas networks based on fluid transient model. J. Energy Resour. Technol. 1995, 117, 324-328. [CrossRef]

18. Kiuchi, T. An implicit method for transient gas flows in pipe networks. Int. J. Heat Fluid Flow 1994, 15, 378-383. [CrossRef]

19. Wylie, E.B.; Stoner, M.A.; Streeter, V.L. Network: System transient calculations by implicit method. Soc. Pet. Eng. J. 1971, 11, 356-362. [CrossRef]

20. Li, Y.; Yao, G. Design and Operation of Gas Pipeline; China University of Petroleum Press: Beijing, China, 2009; pp. 19-26. (In Chinese)

21. Abbaspour, M.; Chapman, K.S. Non-isothermal transient flow in natural gas pipeline. J. Appl. Mech. 2008, 75, 031018. [CrossRef]

22. Luskin, M. An approximation procedure for non-symmetric, nonlinear hyperbolic systems with integral boundary conditions. Siam J. Numer. Anal. 1979, 16, 145-164. [CrossRef]

23. Zheng, J.G.; Chen, G.Q.; Song, F.; Ai, M.Y.; Zhao, J.L. Research on simulation model and solving technology of large scale gas pipe network. J. Syst. Simul. 2012, 14, 133. (In Chinese)

24. Barley, J. Thermal decoupling: An investigation. In Proceedings of the PSIG annual Meeting, Pipeline Simulation Interest Group, Santa Fe, NM, USA, 16-19 April 2012.

25. Helgaker, J.F.; Ytrehus, T. Coupling between continuity/momentum and energy Equation in 1D gas flow. Energy Procedia 2012, 26, 82-89. [CrossRef]

26. Wang, P.; Yu, B.; Deng, Y.; Zhao, Y. Comparison study on the accuracy and efficiency of the four forms of hydraulic Equations of a natural gas pipeline based on linearized solution. J. Nat. Gas Sci. Eng. 2015, 22, 235-244. [CrossRef]

27. Stoner, M.A. Sensitivity analysis applied to a steady-state model of natural gas transportation systems. Soc. Pet. Eng. J. 1972, 12, 115-125. [CrossRef]

28. Wang, P.; Yu, B.; Han, D.; Sun, D. Fast method for the hydraulic simulation of natural gas pipeline networks based on the divide-and-conquer approach. J. Nat. Gas Sci. Eng. 2018, 50, 55-63. [CrossRef]

29. Keenan, P.T. Collation and upwinding for thermal flow in pipelines: The linearized case. Int. J. Numer. Methods Fluids 1996, 22, 835-849. [CrossRef]

30. Osiadacz, A.J.; Chaczykowski, M. Comparison of isothermal and non-isothermal pipeline gas flow models. Chem. Eng. J. 2001, 81, 41-51. [CrossRef] 
31. Wang, P.; Yu, B.; Han, D.; Li, J.; Sun, D.; Xiang, Y.; Wang, L. Adaptive implicit finite difference method for natural gas pipeline transient flow. Oil Gas Sci. Technol. Rev. D'ifp Energ. Nouv. 2018, 73, 21. [CrossRef]

32. Chaczykowski, M. Sensitivity of Pipeline Gas Flow Model to the Selection of the Equation of State. Chem. Eng. Res. Des. 2009, 87, 1596-1603. [CrossRef]

33. Benedict, M.; Webb, G.B.; Rubin, L.C. An empirical Equation for thermodynamic properties of light hydrocarbons and their mixtures I. Methane, ethane, propane and n-butane. J. Chem. Phys. 1940, 8, 334-345. [CrossRef]

34. Scott, L.R. Numerical Analysis, 2nd ed.; Princeton University Press: Oxford, MS, USA, 2016; pp. 53-64, 145-149.

35. Colebrook, C.F. Turbulent flow in pipes with particular reference to the transition region between the smooth and rough pipe laws. J. Inst. Civ. Eng. 1939, 11, 133-156. [CrossRef]

(C) 2019 by the authors. Licensee MDPI, Basel, Switzerland. This article is an open access article distributed under the terms and conditions of the Creative Commons Attribution (CC BY) license (http://creativecommons.org/licenses/by/4.0/). 